\title{
Implementation and Computation of a Value for Generalized Characteristic Function Games
}

\author{
TOMASZ P. MICHALAK, University of Oxford and University of Warsaw \\ PIOTR L. SZCZEPAŃSKI, Warsaw University of Technology and Hewlett-Packard GD Poland \\ TALAL RAHWAN, Masdar Institute of Science and Technology \\ AGATA CHROBAK, University of Warsaw \\ SIMINA BRÂNZEI, Aarhus University \\ MICHAEL WOOLDRIDGE, University of Oxford \\ NICHOLAS R. JENNINGS, University of Southampton and King Abdulaziz University
}

\begin{abstract}
Generalized characteristic function games are a variation of characteristic function games, in which the value of a coalition depends not only on the identities of its members, but also on the order in which the coalition is formed. This class of games is a useful abstraction for a number of realistic settings and economic situations, such as modeling relationships in social networks. To date, two main extensions of the Shapley value have been proposed for generalized characteristic function games: the Nowak-Radzik [1994] value and the Sánchez-Bergantiños [1997] value. In this context, the present article studies generalized characteristic function games from the point of view of implementation and computation. Specifically, the article makes two key contributions. First, building upon the mechanism by Dasgupta and Chiu [1998], we present a non-cooperative mechanism that implements both the Nowak-Radzik value and the SánchezBergantiños value in Subgame-Perfect Nash Equilibria in expectations. Second, in order to facilitate an efficient computation supporting the implementation mechanism, we propose the Generalized MarginalContribution Nets representation for this type of game. This representation extends the results of Ieong and Shoham [2006] and Elkind et al. [2009] for characteristic function games and retains their attractive computational properties.
\end{abstract}

Categories and Subject Descriptors: J.4 [Social and Behavioral Sciences]

General Terms: Algorithms, Economics, Theory

Additional Key Words and Phrases: Generalized characteristic function games, Shapley value, implementation, representation

Tomasz P. Michalak and Michael Wooldridge were supported by the European Research Council under Advanced Grant 291528 ("RACE").

Piotr L. Szczepański and Tomasz P. Michalak were supported the Polish National Science grant DEC2013/09/D/ST6/03920.

Nicholas R. Jennings was supported by the ORCHID Project, funded by an EPSRC (Engineering and Physical Research Council) under the grant EP/I011587/1.

Simina Brânzei was supported by the Sino-Danish Center for the Theory of Interactive Computation, funded by the Danish National Research Foundation and the National Science Foundation of China (under the grant 61061130540). Simina also acknowledges support from the Center for research in the Foundations of Electronic Markets (CFEM), supported by the Danish Strategic Research Council.

Authors' addresses: T. P. Michalak, Department of Computer Science, University of Oxford, UK, and Institute of Informatics, University of Warsaw, Poland; M. Wooldridge, Department of Computer Science, University of Oxford, UK; P. L. Szczepański, Institute of Informatics, Warsaw University of Technology, Poland, and Hewlett-Packard GD Poland; A. Chrobak, Institute of Informatics, University of Warsaw, Poland; S. Brânzei, Aarhus University, Denmark; T. Rahwan, Masdar Institute of Science and Technology, UAE; N. R. Jennings, School of Electronics and Computer Science, University of Southampton, UK, and Department of Computing and Information Technology, King Abdulaziz University, Saudi Arabia. Correspondence email: tomasz. michalak@cs.ox.ac.uk.

Permission to make digital or hard copies of all or part of this work for personal or classroom use is granted without fee provided that copies are not made or distributed for profit or commercial advantage and that copies bear this notice and the full citation on the first page. Copyrights for components of this work owned by others than ACM must be honored. Abstracting with credit is permitted. To copy otherwise, or republish, to post on servers or to redistribute to lists, requires prior specific permission and/or a fee. Request permissions from permissions@acm.org.

(c) 2014 ACM $2167-8375 / 2014 / 10-A R T 16 \$ 15.00$

DOI: http://dx.doi.org/10.1145/2665007 


\section{ACM Reference Format:}

Tomasz P. Michalak, Piotr L. Szczepański, Talal Rahwan, Agata Chrobak, Simina Brânzei, Michael Wooldridge, and Nicholas R. Jennings. 2014. Implementation and computation of a value for generalized characteristic function games. ACM Trans. Econom. Comput. 2, 4, Article 16 (October 2014), 35 pages.

DOI: http://dx.doi.org/10.1145/2665007

\section{INTRODUCTION}

Coalitional games are an important model for many realistic economic situations that capture the ability of players to take joint, coordinated actions. Typically, a coalitional game model specifies payoffs attainable by various subsets (or coalitions) of players cooperating within the game. Given these payoffs, fundamental game-theoretic research questions concerning coalitional games include: (i) Which coalition will actually form? (ii) How should the coalitional payoff be distributed among coalition members? Moreover, assuming that desirable coalitions and payoff distribution methods exist: (iii) How can we create a mechanism that implements a specific solution in an environment of self-interested players? Coalitional games also raise many important questions from the computer-science perspective, key among them being: (iv) How to represent games compactly? and (v) How to efficiently compute their solutions given such compact representations [Chalkiadakis et al. 2011]. This article addresses questions (iii), (iv), and (v) for coalitional games in the generalized characteristic function form as introduced by Nowak and Radzik [1994]. This class of games generalizes characteristic function games with transferable utility by distinguishing between different orders in which players create coalitions. Thus, in this model, the value of a coalition depends not only on its members, but also on the order in which those members joined the coalition.

Generalized characteristic function form games naturally capture a number of realworld situations. Consider, for example, a search on a social network where we need to answer a question that only a few nodes can answer, and the question is propagated through referrals along the connections of each node. This was the case, for instance, with the recent TAG challenge [Rutherford et al. 2013; Rahwan et al. 2013], where photos of five suspects were announced on a particular date, along with the name of the city where each criminal was located, and the challenge was to take photos of as many suspects as possible within 12 hours using referrals on social networks. In such cases, the order in which nodes are added to the search influences the time required to find an answer (e.g., the sooner the nodes with more connections join, the faster the search becomes). As a second example, consider the cost-allocation problem studied by Sánchez and Bergantiños [1999], where a group of universities participating in a joint research project invite a foreign expert for a visit. The budget of such a visit will depend on the planned route, that is, the sequence in which the universities are to host the researcher.

Clearly, situations such as these cannot be captured within a conventional coalitional game model (i.e., a game in characteristic function form), where the value of a coalition depends solely on the identity of its members, without considering the order in which the members have joined it. Consequently, a growing body of work has considered generalized characteristic function games. In this context, a number of researchers have focused on the issue of fair payoff division. The most well-known fair payoff division concept in coalitional games is the Shapley value [Shapley 1953]. The basic idea is that $a_{i}$ 's payoff should be equal to $a_{i}$ 's average marginal contribution, taken over all possible ways in which players could join the game (and contribute to the creation of each coalition's value). For instance, in the game of three players, there are altogether 3 ! ways in which players could join the game: (i) $a_{1}, a_{2}, a_{3}$, (ii) $a_{1}, a_{3}, a_{2}$, (iii) $a_{2}, a_{1}, a_{3}$, (iv) $a_{3}, a_{1}, a_{2}$, (v) $a_{2}, a_{3}, a_{1}$, and (vi) $a_{3}, a_{2}, a_{1}$. As such, there are 3 ! corresponding 
marginal contributions of $a_{1}$ : in (i) and (ii) $a_{1}$ joins first (i.e., $a_{1}$ contributes twice to the empty set); in (iii) $a_{1}$ joins after $a_{2}$ (i.e., $a_{1}$ contributes once to $\left\{a_{2}\right\}$ ); in (iv) $a_{1}$ joins after $a_{3}$ (i.e., $a_{1}$ contributes once to $\left\{a_{3}\right\}$ ); finally in (v) and (vi) $a_{1}$ joins last (i.e., $a_{1}$ contribute twice to $\left.\left\{a_{2}, a_{3}\right\}\right)$. The average of all these marginal contributions is the Shapley value of $a_{1}$.

There are two main extensions of the Shapley value to generalized characteristic function games. The first was proposed by Nowak and Radzik [1994] (which we refer to as the NR value), while the second was introduced by Sánchez and Bergantiños [1997] (which we refer to as the $S B$ value). The difference between these values can be seen in cases (v) and (vi) of the above 3-agent example. In particular, if $v$ denotes the characteristic function, and $v_{g}$ denotes the generalized characteristic function, then:

-with the Shapley value, $a_{1}$ contributes twice the difference between $v\left(\left\{a_{2}, a_{3}\right\}\right)$ and $v\left(\left\{a_{1}, a_{2}, a_{3}\right\}\right)$;

-with the NR value, $a_{1}$ contributes once the difference between $v_{g}\left(a_{2}, a_{3}\right)$ and $v_{g}\left(a_{2}, a_{3}, a_{1}\right)$, and contributes once the difference between $v_{g}\left(a_{3}, a_{2}\right)$ and $v_{g}\left(a_{3}, a_{2}, a_{1}\right)$;

-with the SB value, $a_{1}$ contributes twice the difference between the average value of a coalition consisting of $a_{2}, a_{3}$ and of a coalition consisting of $a_{1}, a_{2}, a_{3}$, that is, difference between $\frac{v_{g}\left(a_{2}, a_{3}\right)+v_{g}\left(a_{3}, a_{2}\right)}{2}$ and $\frac{v_{g}\left(a_{1}, a_{2}, a_{3}\right)+v_{g}\left(a_{1}, a_{3}, a_{2}\right)+v_{g}\left(a_{2}, a_{1}, a_{3}\right)+v_{g}\left(a_{2}, a_{3}, a_{1}\right)+v_{g}\left(a_{3}, a_{1}, a_{2}\right)+v_{g}\left(a_{3}, a_{2}, a_{1}\right)}{6}$.

One of the interesting applications of those two extensions is the recent body of literature that uses game theoretic solution concepts to compute centralities of nodes in networks [del Pozo et al. 2011; Amer et al. 2007, 2010]. In summary, by defining a coalitional game with players being nodes of a network, and then computing a solution for such a game, it is possible to obtain a measure of importance for individual nodes. In this context, both the NR and SB values were used by del Pozo et al. [2011] to study the centrality of nodes in directed social networks. These networks have recently raised increasing attention as they can be used to model a variety of situations, ranging from terrorist groups [Brams et al. 2006] to the spread of contagious diseases [Lentz et al. 2012]. The crucial characteristics of these real-life network applications is that a relationship between two nodes connected by an edge is asymmetric, that is, the edge is directed. Consequently, in many cases, the worth of a coalition in a game defined over such a network should depend not only on its members but also on the order in which they were incorporated to this coalition. Del Pozo et al. [2011] took this into account by defining a generalized characteristic function game over a network (instead of a characteristic function game) and considering both NR and SB values as centrality measures.

Although there have been a number of game-theoretic works (including the NR and SB values) on generalized characteristic function games, the implementational and the computational aspects of these games have not been yet studied. Both research challenges can be summarized as follows:

-Implementation. Given a desired solution to a coalitional game (such as the Shapley value), the issue of implementation deals with creating a set of rules (a mechanism) that incentivizes self-interested players to reach the desired solution as a result of equilibrium behavior. Although there exist various mechanisms implementing the Shapley value and some of its various extensions, no mechanism for coalitional games with ordered coalitions has been proposed to date.

-Computation. One of the key questions in generalized characteristic function games is how to represent these games compactly. Clearly, a straightforward listing of the values for all possible ordered coalitions requires space of size hyper-exponential in the number of players. In contrast, a well-crafted representation may not only 
facilitate abstract reasoning but also may be able to capture certain patterns more concisely, and may facilitate the development of significantly more efficient solutions to challenging computational problems. Although a number of representations have been proposed in the computer-science and AI communities for various classes of games [Deng and Papadimitriou 1994; Ohta et al. 2006, 2009; Conitzer and Sandholm 2006; Greco et al. 2009; Ieong and Shoham 2006; Michalak et al. 2009], no representation has been developed to date that facilitates faster computation of the NR and SB values for generalized characteristic function games.

In order to apply a solution to a coalitional game in practice, it is necessary that both of these two aspects (i.e., implementation and computation) are considered jointly (see, e.g., Lehmann et al. [2002]). Indeed, the ability to compute the solution is of no use if the players are not incentivized to use that solution in the first place. Similarly, having the players agree to use a solution that cannot be efficiently computed is deprecating its importance (in such cases, the players are forced to use approximation algorithms that bring the unsolicited portion of uncertainty into their actions).

Against this background, this article is the first study of generalized characteristic function games from the implementational and computational points of view. In particular:

-We present a mechanism that implements the NR value and the SB value in expectations. Our mechanism is an extension of the mechanism by Dasgupta and Chiu [1998], called Simple Demand Commitment Games, which implements the Shapley value given that the characteristic function is strictly convex. We call our extension Ordered Demand Commitment Games.

-We present a logic-based representation for generalized characteristic function games. Our representation builds upon the marginal contribution nets (MC-Nets) representation of Ieong and Shoham [2006] and its extension by Elkind et al. [2009]. MC-Nets have proven to be very efficient with respect to some important computational problems [Ieong and Shoham 2006; Michalak et al. 2010; Ohta et al. 2009], especially the computation of the Shapley value. We demonstrate that our representation (which builds upon MC-Nets) is fully expressive, that is, it is able to represent any generalized coalitional game. We also show that, for certain games, our representation is exponentially more concise than the generalized function game representation and that it can be used to efficiently compute both the NR value and the SB value. Finally, as a sample application, we show how our representation facilitates computations of game-theoretic betweeness centrality for directed networks based on the NR and SB values.

The remainder of the article is organized as follows. In Section 2, we discuss the related literature. Section 3 provides notation and formal definitions of the Shapley value and its two extensions to generalized characteristic function games (i.e., the NR and SB values). In Section 4, we describe our mechanism for implementing both values. The new representation is introduced and analyzed in Section 5. Conclusions follow.

\section{RELATED WORK}

In this section, we discuss three bodies of literature related to our work: (i) the literature on noncooperative implementations of the Shapley value and its various extensions; (ii) the computer-science literature on concise representations of coalitional games; and (iii) the literature on further extensions of the Shapley value for generalized characteristic function games. 
The issue of implementing the Shapley value has been studied in the literature by a number of authors. Gul [1989] introduced a model of a transferable-utility economy where two players make bilateral offers at random meetings. Assuming that the game is strictly convex, with the time interval between meetings becoming arbitrarily small, the Shapley value emerges as the limit for the expected payoff of each player in a Stationary Subgame-Perfect Nash Equilibrium (SSPNE). This result holds also for strictly superadditive games but only for the SSPNE reached by an immediate agreement [Gul 1989; Hart and Levy 1999]. A simplified mechanism of this kind was introduced by Evans [1996].

An alternative mechanism, called the Simple Demand Commitment Game (SDCG), was later on introduced by Dasgupta and Chiu [1998]. In this article, we will extend SDCG to the generalized characteristic functions setting.

Assuming that the characteristic function is strictly convex, SDCG starts by randomly choosing an order in which players are allowed to move. Then, the first player in the chosen order makes a move which may or may not end the game. If the game does not end, then the second player in the order makes another move (which again may or may not end the game), and so on. In particular, the move that each player $a_{i}$ makes is to select one of the following two options: (1) demand a payoff $d_{i}$ that $a_{i}$ will accept in return for joining any coalition, or (2) create a coalition consisting of $a_{i}$ and a (possibly empty) subset of his choice out of the players that precede him in the order, which automatically ends the game and forces every nonmember of that coalition to form a singleton coalition.

Pérez-Castrillo and Wettstein [2001] proposed a mechanism that has some features strictly better than the SDCG. In particular, while SDCG implements the Shapley value in expected terms, in the mechanism by Pérez-Castrillo and Wettstein, the Shapley value emerges in all equilibria. Furthermore, compared to Dasgupta and Chiu's mechanism, which requires strict convexity, Pérez-Castrillo and Wettstein's mechanism requires the characteristic function to be zero-monotonic, which is a weaker condition. In more detail, the mechanism by Pérez-Castrillo and Wettstein involves three steps. In Step 1, players bid by offering each other transfers, and the stake is to become a proposer, that is, a player that has the sole right to divide the payoff from the game. In Step 2, the winner (i.e., the highest net bidder) pays the transfers promised to other players, and then proposes a division of the game's payoff among the players in the game. In Step 3, these players either accept or reject the proposal. If the offer is rejected, the proposer is obliged to leave the mechanism and form a singleton coalition. The remaining players follow the same procedure but for the now-smaller game.

A number of follow-up works have built upon Pérez-Castrillo and Wettstein's mechanism. In particular, a version of the mechanism that implements the Ordinal Shapley value [Pérez-Castrillo and Wettstein 2006] for $n=3$ was proposed by Pérez-Castrillo and Wettstein [2005]. To implement the Owen value, Vidal-Puga and Bergantiños [2003] added a fourth step-a bidding phase to become leaders of a priori given coalitions. A different fourth step was proposed by $\mathrm{Ju}$ and Wettstein [2007] in order to implement the extension of the Shapley value to games with externalities by Pham do and Norde [2007]. A similar approach followed in Macho-Stadler et al. [2006, 2010]. Van den Brink and Funaki [2010] introduced a discounting parameter to implement the discounted Shapley value.

The common and crucial feature of all the values that were implemented in the spirit of the mechanism by Pérez-Castrillo and Wettstein is that they all meet a property called the balanced contribution. Essentially, this property states that for any two players, $a_{i}, a_{j}$, the worth of $a_{i}$ to $a_{j}$ is the same as the worth of $a_{j}$ to $a_{i}$. More formally, 
focusing our attention on the Shapley value: ${ }^{1}$

$$
\phi_{i}(N, v)-\phi_{i}\left(N_{-j}, v\right)=\phi_{j}(N, v)-\phi_{j}\left(N_{-i}, v\right),
$$

where $\phi_{i}(N, v)$ denotes the Shapley value of player $a_{i}$ in the coalitional game with player set $N$ and value function $v$, while, $\phi_{i}\left(N_{-i}, v\right)$ denotes the Shapley value of player $a_{i}$ in the coalitional game with player set $N \backslash\left\{a_{i}\right\}$ and the same value function $v$.

Pérez-Castrillo and Wettstein [2005] showed how that the balanced contribution property allows for the construction of a mechanism in which the Shapley value emerges as a result of equilibrium behavior. Unfortunately, it can be very easily demonstrated that the balanced contribution property is not met by the NR value for games in the generalized characteristic function form:

Example 2.1. Let $N=\left\{a_{1}, a_{2}\right\}$ and $v_{g}\left(\left(a_{1}\right)\right)=1, v_{g}\left(\left(a_{2}\right)\right)=2, v_{g}\left(\left(a_{1}, a_{2}\right)\right)=3$, and $v_{g}\left(\left(a_{2}, a_{1}\right)\right)=8$. To compute the Nowak and Radzik value, we average contributions of the players to ordered coalitions over all permutations of players, that is over $\left(a_{1}, a_{2}\right)$ and $\left(a_{2}, a_{1}\right)$. As a result of this computation, we find that the Nowak and Radzik value of the first player is: $\phi_{1}^{N R}(N)=\frac{(1-0)+(8-2)}{2}=3.5$, and of the second players is: $\phi_{2}^{N R}(N)=\frac{(2-0)+(3-1)}{2}=2$. Finally, it is easy to see that: $\phi_{1}^{N R}\left(N_{-2}\right)=1$, and $\phi_{2}^{N R}\left(N_{-1}\right)=2$.

Turning now to the computer-science literature on developing concise representations of coalitional games, Wooldridge and Dunne [2006] divide such representations into two broad categories. The first category gives the characteristic function a specific interpretation in terms of combinatorial structures such as graphs. This is, for instance, the approach adopted by Deng and Papadimitriou [1994], Greco et al. [2009], and Wooldridge and Dunne [2006], and the advantage is that such representations are guaranteed to be succinct. However, the disadvantage is that they are not always fully expressive. The second category of representations is designed with the emphasis on full expressivity at the expense of not being always succinct. In addition to MC-Nets [Ieong and Shoham 2006; Elkind et al. 2009] and its various extensions [Michalak et al. 2010 ] to which our article also contributes, the second category includes the SynergyCoalition-Groups representation [Conitzer and Sandholm 2006; Ohta et al. 2009] and the Algebraic-Decision-Diagrams representation [Aadithya et al. 2011; Sakurai et al. 2011]. A comprehensive discussion on representation formalisms for various classes of coalitional games can be found in Chalkiadakis et al. [2011].

Although in this article we focus on exact computations, we note that there exist a few approximation methods for the Shapley value and, potentially, some of them can be extended to the NR and SB values. While most of such methods concern certain specific subclasses of characteristic function games [Fatima et al. 2008; Bachrach et al. 2008], the Monte Carlo approximation algorithm by Castro et al. [2009] can be efficiently applied to all characteristic function games, provided that the worth of every coalition can be computed in polynomial time. More specifically, instead of computing the average marginal contribution of each player over all permutation (as in the definition of the Shapley value), the algorithm approximates this average by considering only a subset of permutations, sampled uniformly from the space of permutations. The approximation error can be bounded, for example, if the variance of marginal contributions is known. We believe this algorithm can be easily extended to approximate both the NR value and the SB value. In particular, the algorithm should be modified such that, for each sampled permutation, it computes the marginal contribution of each player to the ordered coalition that precedes it in the permutation. For the NR value, this marginal

\footnotetext{
${ }^{1}$ Formula (1) remains the same for all the extensions of the Shapley value implemented in the spirit of Pérez-Castrillo and Wettstein.
} 
contribution is awarded to the player that has joined the ordered coalition, while for the SB value, the marginal contribution is distributed equally among the player and the members of the ordered coalition it has joined. Such approximation algorithms can be used in situations in which the game cannot be easily represented using our generalized read-once MC-Nets formalism.

Finally, we mention other extensions of the Shapley value for generalized characteristic function games developed after the Nowak-Radzik and Sánchez-Bergantiños values. In particular, a family of weighted Shapley values was studied by Bergantiños and Sánchez [2001]. Furthermore, a parametric family of values (including both the Nowak-Radzik and Sánchez-Bergantiños values) was analyzed in a network context by del Pozo et al. [2011].

\section{PRELIMINARIES}

We begin by describing the basic notation (Appendix B provides a comprehensive summary). Let $N=\left\{a_{1}, \ldots, a_{n}\right\}$ be the set of players in a coalitional game. Denote by $2^{N}$ the set of all subsets of $N$. An element of $2^{N}$ is a coalition. An arbitrary coalition will often be denoted $C$ or $D$. The coalition involving all players in the game will be called the grand coalition. A characteristic function $v$ is a mapping $v: 2^{N} \rightarrow \mathbb{R}$, that is, it assigns to every coalition $C \subseteq N$ a real number representing its value. We will assume that $v(\emptyset)=0$. A game in characteristic function form is a pair $(N, v)$. When there is no risk of confusion, we will sometimes simply write $v$ instead of $(N, v)$.

For each coalition $C \in 2^{N} \backslash\{\emptyset\}$, denote by $\Pi(C)$ the set of all possible permutations of the players in $C$. Any such permutation will be called an ordered coalition. An arbitrary ordered coalition will often be denoted as $T$ or $S$, while the set of all such coalitions will be denoted $\mathcal{T}$. That is, $\mathcal{T}=\bigcup_{C \in 2^{N}} \Pi(C)$. A generalized characteristic function $v_{g}$ is a mapping $v_{g}: \mathcal{T} \rightarrow \mathbb{R}$, where it is assumed that $v_{g}(\emptyset)=0$. A game in generalized characteristic function form is a tuple $\left(N, v_{g}\right)$, and will sometimes be denoted by $v_{g}$ alone. For some coalition $D \subseteq N$ we will denote by $\mathcal{T}_{-D}$ the set of all ordered coalitions not containing players from $\bar{D}$, formally: $\mathcal{T}_{-D}=\bigcup_{C \in 2^{N \backslash D}} \Pi(C)$. Sometimes, we will write $a_{i}$ instead of $\left(a_{i}\right)$ for brevity.

We will sometimes refer to the members of an ordered coalition $T$ using their names, for example, write $T=\left(a_{5}, a_{2}, a_{3}\right)$, while other times we may refer to them using a lower case of the same letter: $T=\left(t_{1}, \ldots, t_{|T|}\right)$, meaning that $t_{i}$ is the $i$ th agent in $T$. Furthermore, given two disjoint ordered coalitions, $T=\left(t_{1}, \ldots, t_{|T|}\right) \in \mathcal{T}$ and $S=\left(s_{1}, \ldots, s_{|S|}\right) \in \mathcal{T}$, we write $(T, S)^{k}$ to denote the ordered coalition that results from inserting $S$ at the $k$ th position in $T$. That is, $(T, S)^{k}=\left(t_{1}, \ldots, t_{k-1}, s_{1}, \ldots, s_{|S|}, t_{k}, \ldots, t_{|T|}\right)$. With a slight abuse of notation, we write $\left(T, a_{i}\right)^{k}$ to denote $\left(T,\left(a_{i}\right)\right)^{k}$. Furthermore, we write $\left(a_{i}, T\right)$, and $\left(T, a_{i}\right)$, to denote the ordered coalition that results from inserting $a_{i}$ to $T$ as the first player, and the last player, respectively.

For every coalition $C \subseteq N$ and every permutation $\pi=\left\{\pi_{1}, \pi_{2}, \ldots, \pi_{|\pi|}\right\} \in \Pi(C)$, we introduce a function $\operatorname{inv}(\bar{\pi})$ that returns the inverse of $\pi$. Formally, inv : $\bigcup_{C \subseteq N} \Pi(C) \rightarrow$ $\bigcup_{C \subseteq N} \Pi(C)$ is given by: $i n v(\pi)=\left(\pi_{|\pi|}, \ldots, \pi_{2}, \pi_{1}\right)$. For instance, for $\pi=\left(a_{3}, a_{1}, a_{5}, a_{6}\right)$, we have $\operatorname{inv}(\pi)=\left(a_{6}, a_{5}, a_{1}, a_{3}\right)$. Furthermore, given a permutation $\pi \in \Pi(N)$ and a coalition $C \subseteq N$, with a slight abuse of notation, we will denote by $\pi(C)$ the ordered coalition consisting of all the players in $C$ ordered according to $\pi$, that is, it is the ordered coalition that results after removing from $\pi$ every player in $N \backslash C$. For example, given $\pi=$ $\left(a_{2}, a_{1}, a_{4}, a_{3}\right)$, and $C=\left\{a_{1}, a_{2}, a_{3}\right\}$, we have $\pi(C)=\left(a_{2}, a_{1}, a_{3}\right)$. Moreover, given a generalized game $\left(N, v_{g}\right)$, and a permutation $\pi \in \Pi(N)$, we denote by $\left(N, v_{g, \pi}\right)$ the characteristic function game in which, $\forall C \subseteq N$ :

$$
v_{g, \pi}(C)=v_{g}(\pi(C))
$$


For any $\left(N, v_{g}\right)$, we also introduce the characteristic function game $\left(N, \bar{v}_{g}\right)$ which we call the average game of $\left(N, v_{g}\right)$. In this game, $\forall C \subseteq N$ :

$$
\bar{v}_{g}(C)=\frac{1}{|\Pi(C)|} \sum_{T \in \Pi(C)} v_{g}(\pi(C)) .
$$

We will call $\bar{v}_{g}$, the average characteristic function of $\left(N, v_{g}\right)$.

Next, we extend the notion of a subset to ordered sets.

Definition 3.1. For any two ordered coalitions $S=\left(s_{1}, \ldots, s_{|S|}\right) \in \mathcal{T}$ and $T=$ $\left(t_{1}, \ldots, t_{|T|}\right) \in \mathcal{T}$, we say that $T$ is a subset of $S$, and write $T \subseteq S$, if and only if $T$ is a subsequence of $S$, that is, the following two conditions hold.

-Every member of $T$ is a member of $S$. More formally:

$$
\forall t_{i} \in T, \quad \exists s_{k} \in S: s_{k}=t_{i} .
$$

-For any two players, $t_{i}, t_{j} \in T$, if $t_{i}$ appears before $t_{j}$ in $T$, then $t_{i}$ also appears before $t_{j}$ in $S$. More formally:

$$
\forall t_{i}, t_{j} \in T: i<j, \exists s_{k}, s_{w} \in S: k<w \text { and } s_{k}=t_{i} \text { and } s_{w}=t_{j} .
$$

Following convention, we say that $T$ is a strict subset of $S$, and write $T \vec{\subset} S$ (instead of $T \subseteq S$ ), if these two conditions are met, and $T \neq S$.

Now, we are ready to introduce the following definition:

Definition 3.2. A characteristic function game $(N, v)$ is said to be convex if, for every two coalitions $C, D: D \subset C$ and for every $a_{i} \in N \backslash C$, the following holds:

$$
v\left(C \cup\left\{a_{i}\right\}\right)-v(C) \geq v\left(D \cup\left\{a_{i}\right\}\right)-v(D) .
$$

Furthermore, $(N, v)$ is said to be strictly convex if:

$$
v\left(C \cup\left\{a_{i}\right\}\right)-v(C)>v\left(D \cup\left\{a_{i}\right\}\right)-v(D) .
$$

We extend the notion of convexity to the generalized characteristic function games as follows.

Definition 3.3. A generalized characteristic function game $\left(N, v_{g}\right)$ is said to be convex if, for every two ordered coalitions $S, T: T \vec{\subset} S$ and for every $a_{i} \in N \backslash S$, we have

$$
v_{g}\left(\left(S, a_{i}\right)^{s}\right)-v_{g}(S) \geq v_{g}\left(\left(T, a_{i}\right)^{t}\right)-v_{g}(T)
$$

whenever $\left(T, a_{i}\right)^{t}$ is a subset of $\left(S, a_{i}\right)^{s}$. More formally, the inequality holds for every $t \in\{1, \ldots,|T|+1\}, s \in\{1, \ldots,|S|+1\}:\left(T, a_{i}\right)^{t} \vec{\subset}\left(S, a_{i}\right)^{s}$. Furthermore, $\left(N, v_{g}\right)$ is said to be strictly convex if

$$
v_{g}\left(\left(S, a_{i}\right)^{s}\right)-v_{g}(S)>v_{g}\left(\left(T, a_{i}\right)^{t}\right)-v_{g}(T)
$$

Now, we briefly describe the Shapley value for characteristic function games, and then present its extensions to generalized characteristic function games. The Shapley value was proposed as a normative scheme for dividing the value of the game fairly among the players. In more detail, the Shapley value of a player $a_{i} \in N$, denoted $\phi_{i}(N, v)$, is $a_{i}$ 's share of the grand coalition's payoff, which is computed as the average marginal contribution of that player over all possible joining orders (assuming that the agents have joined the game sequentially, one agent at a time). Formally:

$$
\phi_{i}(N, v)=\frac{1}{|N| !} \sum_{\pi \in \Pi(N)} \Delta_{v}\left(C_{\overleftarrow{\pi_{i}}}, a_{i}\right)
$$


where $\Delta_{v}\left(C_{\overleftarrow{\pi_{i}}}, a_{i}\right)$ is the marginal contribution of a player $a_{i}$ to a coalition $C_{\overleftarrow{\pi_{i}}}$ consisting of all the players that are in permutation $\pi$ before $a_{i}$. Formally:

$$
\Delta_{v}\left(C_{\overleftarrow{\pi_{i}}}, a_{i}\right)=v\left(C_{\overleftarrow{\pi_{i}}} \cup\left\{a_{i}\right\}\right)-v\left(C_{\overleftarrow{\pi_{i}}}\right)
$$

Importantly, as visible from Eq. (4), if $\pi \in \Pi(N)$ was selected uniformly at random, the Shapley value of player $a_{i}$ would be the expected marginal contribution of $a_{i}$ to $C_{\overleftarrow{\pi_{i}}}$. That is, $\phi_{i}(N, v)=\mathbb{E}\left[\Delta_{C_{\tilde{\pi}_{i}}, i}\right]$, where $\mathbb{E}[\cdot]$ is the expectation operator.

It is possible to rewrite Eq. (4) as follows:

$$
\phi_{i}(N, v)=\sum_{\pi \in N \backslash\left\{a_{i}\right\}} \frac{(|N|-|C|-1) !(|C| !)}{|N| !} v\left(C \cup\left\{a_{i}\right\}\right)-v(C) .
$$

This is more computationally efficient than Eq. (4), because the sum is over coalitions, not permutations. When there is no risk of confusion, instead of $\phi_{i}(N, v)$, we will write $\phi_{i}(v)$ or $\phi_{i}$ for brevity. This also concerns the extensions of the Shapley value that will be presented later on in this section.

The Shapley value is "fair" in the sense that it is the unique solution that has the following axioms.

Symmetry. The payoffs do not depend on the players' names. That is, $\phi(\pi(v))=\pi(\phi)(v)$ for every game $v$ and permutation $\pi \in \Pi(N)$.

Null Player. The players that make no contribution should receive nothing. In other words, we have $\left(\forall C \subseteq N, \Delta_{v}\left(C_{\overleftarrow{\pi_{i}}}, a_{i}\right)=0\right) \Rightarrow\left(\phi_{i}(N)=0\right)$.

Efficiency. The entire payoff of the grand coalition should be distributed among its members. That is, $\sum_{a_{i} \in N} \phi_{i}(N)=v(N)$.

Additivity. Given three games, $\left(N, v_{1}\right),\left(N, v_{2}\right)$ and $\left(N, v_{3}\right)$, where $v_{1}(C)=v_{2}(C)+$ $v_{3}(C)$, it holds that, for all $C \subseteq N$, the payoff of a player in $\left(N, v_{1}\right)$ is the sum of its payoffs in $\left(N, v_{2}\right)$ and in $\left(N, v_{3}\right)$.

Whereas these four axioms uniquely determine the Shapley value for characteristic function games, the situation is more complex for generalized games, because a player's marginal contribution (and consequently the null-player axiom) depends on where the new player in the coalition is placed. In this respect, Nowak and Radzik [1994] developed an extension of the Shapley value by making perhaps the most natural assumption that the marginal contribution of a player is computed when this player is placed last in the coalition. Let us denote this marginal contribution of $a_{i}$ to $T \in$ $\mathcal{T}\left(N \backslash\left\{a_{i}\right\}\right)$ in game $v_{g}$ (according to Nowak and Radzik's definition) as $\Delta_{v_{g}}^{N R}\left(T, a_{i}\right)$. Then:

$$
\Delta_{v_{g}}^{N R}\left(T, a_{i}\right)=v_{g}\left(\left(T, a_{i}\right)\right)-v_{g}(T)
$$

In what follows, for any ordered coalition, $T$, let $T\left(a_{i}\right)$ denote the sequence of players in $T$ that appear before $a_{i}$ (if $a_{i} \notin T$ then $T\left(a_{i}\right)=T$ ). For example, given $T=\left(a_{1}, a_{3}, a_{4}, a_{6}\right)$, we have $T\left(a_{4}\right)=\left(a_{1}, a_{3}\right)$. Using this notation, the Nowak-Radzik value (or the NR value for short) is defined as follows:

$$
\phi_{i}^{N R}\left(N, v_{g}\right)=\frac{1}{|N| !} \sum_{T \in \Pi(N)} \Delta_{v_{g}}^{N R}\left(T\left(a_{i}\right), a_{i}\right)=\mathbb{E}\left[\Delta_{v_{g}}^{N R}\left(T\left(a_{i}\right), a_{i}\right)\right] .
$$

This can be written differently as follows:

$$
\phi_{i}^{N R}\left(N, v_{g}\right)=\sum_{C \subseteq N_{-i}} \sum_{T \in \Pi(C)} \frac{(|N|-|T|-1) !}{|N| !}\left[v_{g}\left(\left(T, a_{i}\right)\right)-v_{g}(T)\right]
$$

The NR value is the unique value that satisfies the following "fairness" axioms: 
Efficiency. $\sum_{a_{i} \in N} \phi_{i}^{N R}\left(v_{g}\right)=\frac{1}{|N| !} \sum_{T \in \Pi(N)} v_{g}(T)$;

Null-Player. $\forall a_{i} \in N$, if $v_{g}(T)=v_{g}\left(\left(T, a_{i}\right)\right) \forall T \in \mathcal{T}: a_{i} \notin T$, then $\phi_{i}^{N R}\left(v_{g}\right)=0$;

Additivity. $\phi^{N R}\left(v_{g}+v_{g}^{\prime}\right)=\phi^{N R}\left(v_{g}\right)+\phi^{N R}\left(v_{g}^{\prime}\right)$ for any two functions, $v_{g}$ and $v_{g}^{\prime}$.

Sánchez and Bergantiños [1997] developed an alternative extension of the Shapley value based on the definition of the marginal contribution, where, instead of assuming that this player will be placed last, the authors take the average over all possible positions in which the player can be placed:

$$
\Delta_{v_{g}}^{S B}\left(T, a_{i}\right):=\frac{1}{(|T|+1)} \sum_{l=1}^{|T|+1}\left[v_{g}\left(\left(T, a_{i}\right)^{l}\right)-v_{g}(T)\right] .
$$

The Sánchez-Bergantiños value (or $S B$ value for short) is then computed as

$$
\phi_{i}^{S B}\left(N, v_{g}\right)=\frac{1}{|N| !} \sum_{T \in \Pi(N)} \Delta_{v_{g}}^{S B}\left(T, a_{i}\right)=\mathbb{E}\left[\Delta_{T\left(a_{i}\right)}^{S B}\right] .
$$

This also can be rewritten differently as follows:

$$
\phi_{i}^{S B}\left(N, v_{g}\right)=\sum_{C \subseteq N_{-i}} \sum_{T \in \Pi(C)} \frac{(|N|-|T|-1) !}{|N| !(|T|+1)} \sum_{l=1}^{|T|+1}\left[v_{g}\left(\left(T, a_{i}\right)^{l}\right)-v_{g}(T)\right] .
$$

As noted by Sánchez and Bergantiños [1997], their value for $v_{g}$ is equivalent to the Shapley value of the average game of $v_{g}$ (see Eq. (3) for the definition of the average game), that is,

$$
\phi_{i}^{S B}\left(N, v_{g}\right)=\phi_{i}\left(N, \bar{v}_{g}\right)=\mathbb{E}\left[\Delta_{\bar{v}_{g}}\left(C_{\overleftarrow{\pi}_{i}}, a_{i}\right)\right]
$$

The SB value is the unique value that satisfies NR's efficiency and additivity axioms and the following axioms:

Null-Player. If $\forall T \in \mathcal{T} \forall l \in\{1, \ldots,|T|+1\}: v_{g}\left(\left(T, a_{i}\right)^{l}\right)=v_{g}(T)$, then $\phi_{i}^{S B}\left(v_{g}\right)=0$;

Symmetry. If $\forall T \in \mathcal{T}_{-\{i, j\}} \forall l \in\{1, \ldots,|T|+1\} \quad: \quad v_{g}\left(\left(T, a_{i}\right)^{l}\right)=v_{g}\left(\left(T, a_{j}\right)^{l}\right)$, then $\phi_{i}^{S B}\left(v_{g}\right)=\phi_{j}^{S B}\left(v_{g}\right)$.

The difference between the NR and SB values is illustrated in the following example:

Example 3.4. Consider a game with an ordered coalition $T^{*} \in \Pi(N)$ such that $v_{g}(T)=1$ if $T=T^{*}$ and $v_{g}(T)=0$ otherwise. Then, the average value of the grand coalition, taken over all possible orders, which is $\frac{1}{n !}$, needs to be distributed among the players. Using the NR value, we get $\phi_{t}^{N R}(N)=\frac{1}{n !}$, where $a_{t}$ is the last player in the ordered coalition $T^{*}$, and we get $\phi_{i}^{N R}(N)=0$ for all $a_{i} \in N \backslash\left\{a_{t}\right\}$. In contrast, using the SB value, we get $\phi_{i}^{S B}(N)=\frac{1}{n ! \cdot\left|T^{\prime}\right|}=\frac{1}{n ! \cdot n}$ for all $a_{i} \in N$. As can be seen, in this example, the NR value rewards the last player in the order, whereas the SB value rewards all players equally.

Having introduced the Shapley value and its extensions to generalized characteristic function games, in the following section, we consider the issue of implementation. 


\section{IMPLEMENTATION}

Among the many deeply studied aspects of the Shapley value is whether there exists a set of rules (or a mechanism) that incentivizes self-interested players to adopt the Shapley value as a result of equilibrium behavior. ${ }^{2}$ In this section we propose a mechanism to implement the NR and SB values in Subgame-Perfect Nash Equilibria (SPNE). ${ }^{3}$ We build upon the mechanism by Dasgupta and Chiu [1998].

Given a characteristic function game $v$, Dasgupta and Chiu's mechanism is called the Simple Demand Commitment Game, denoted by $S D C G(v)$. The mechanism proposed in this section modifies it to handle ordered coalitions, that is, to handle a generalized characteristic function game $v_{g}$. As such, we call this mechanism Ordered Demand Commitment Game. It has two versions, one for the NR value (called $O D C G^{N R\left(v_{g}\right)}$ ) and the other for the SB value (called $O D C G^{S B}\left(v_{g}\right)$ ). Section 4.1 presents $O D C G^{N R}\left(v_{g}\right)$, while Section 4.2 presents $O D C G^{S B}\left(v_{g}\right)$. Section 4.3 proves that each mechanism implements its respective value, and that each of the aforementioned strategies is, in fact, an Subgame-Perfect Nash Equilibria (SPNE).

\subsection{The $O D C G^{N R}\left(v_{g}\right)$ Mechanism}

The mechanism $O D C G^{N R}\left(v_{g}\right)$ has two main steps.

- Step 1. An order of players is chosen uniformly at random out of all possible orders. Without loss of generality, let the chosen order be $\pi=\left(a_{1}, \ldots, a_{n}\right)$.

-Step 2. The first player in $\pi$ (i.e., $a_{1}$ ) makes the first move, then the second player in $\pi$ (i.e., $a_{2}$ ) makes the second move (unless $a_{1}$ has terminated the game), then the third player in $\pi$ (i.e., $a_{3}$ ) makes the third move (unless the game has been terminated before his turn), and so on. The move of every $a_{i}: 1 \leq i<n$ involves choosing one of the following two options:

-Option 1. Specify a "demand" $d_{i} \in \mathbb{R}$-an amount of utility that $a_{i}$ will accept in return for joining any coalition. The mechanism then proceeds to the subsequent player in the order, that is, $a_{i+1}$.

-Option 2. Select a subset $C \subseteq\left\{a_{1}, \ldots, a_{i-1}\right\}$ that $a_{i}$ wants to join. This terminates the game with the following outcome: Coalition $i n v\left(\pi\left(C \cup\left\{a_{i}\right\}\right)\right)$ forms, and its payoff is divided as follows: Every $a_{k} \in C$ receives $d_{k}$, while $a_{i}$ receives:

$$
v_{g}\left(\operatorname{inv}\left(\pi\left(C \cup\left\{a_{i}\right\}\right)\right)\right)-\sum_{a_{k} \in C} d_{k} .
$$

In other words, $a_{i}$ pays the members their demands, and takes the surplus for himself. As for non-members, every $a_{j} \in N \backslash\left(C \cup\left\{a_{i}\right\}\right)$ is left with no choice but to form the singleton coalition $\left\{a_{j}\right\}$ and receive the payoff $v_{g}\left(\left(a_{j}\right)\right)$.

Player $a_{n}$ on the other hand has only one choice, which is Option 2.

Note that this mechanism is a game of perfect information, as the chosen order is made publicly known before any player makes a move. Step 2 of $O D C G^{N R}\left(v_{g}\right)$ and $O D C G^{S B}\left(v_{g}\right)$ will be denoted by $O D C G_{\pi}^{N R}\left(v_{g}\right)$ and $O D C G_{\pi}^{S B}\left(v_{g}\right)$, where $\pi$ is the order chosen in Step 1.

\footnotetext{
${ }^{2}$ This is part of the Nash program, which tries to provide a non-cooperative foundation for cooperative solution concepts [Serrano 2004].

${ }^{3}$ The SPNE of a game $G$ are all strategy profiles $s$ such that for any subgame $G^{\prime}$ of $G$, the restriction of $s$ to $G^{\prime}$ is a Nash Equilibrium of $G^{\prime}$. For more details, see Shoham and Leyton-Brown [2009].
} 
Table I. A Comparison between the SDCG Mechanism by Dasgupta and Chiu [1998] and Our $O D C G^{N R}\left(v_{g}\right)$ and $\operatorname{ODCG}^{S B}\left(v_{g}\right)$ Mechanisms

\begin{tabular}{|c|c|c|c|c|}
\hline & Option 1 of $a_{i}$ & Option 2 of $\alpha_{i}$ & $\begin{array}{l}\text { Coalition created } \\
\text { by the mechanism }\end{array}$ & Payoffs \\
\hline$S D C G_{\pi}(v)$ & $\begin{array}{c}\text { demand } \\
d_{i}\end{array}$ & $\begin{array}{c}\text { choose a subset } \\
C \in\left\{a_{1}, \ldots, a_{i-1}\right\}\end{array}$ & $C \cup\left\{a_{i}\right\}$ & $\begin{array}{l}\forall a_{k} \in C: p_{k}=d_{k} \\
p_{i}=v\left(C \cup\left\{a_{i}\right\}\right)-\sum_{a_{k} \in C} d_{k} \\
\forall a_{k} \in N \backslash\left\{C \cup\left\{a_{i}\right\}\right\}: p_{k}=v\left(\left\{a_{k}\right\}\right)\end{array}$ \\
\hline$O D C G_{\pi}^{N R}\left(v_{g}\right)$ & $\begin{array}{c}\text { demand } \\
d_{i}\end{array}$ & $\begin{array}{c}\text { choose a subset } \\
C \in\left\{a_{1}, \ldots, a_{i-1}\right\}\end{array}$ & $C \cup\left\{a_{i}\right\}$ ordered as inverse of $\pi$ & $\begin{array}{l}\forall a_{k} \in C: p_{k}=d_{k} \\
p_{i}=v_{g}\left(\operatorname{inv}\left(\pi\left(C \cup\left\{a_{i}\right\}\right)\right)\right)-\sum_{a_{k} \in C} d_{k} \\
\forall a_{k} \in N \backslash\left\{C \cup\left\{a_{i}\right\}\right\}: p_{k}=v_{g}\left(\left(a_{k}\right)\right) .\end{array}$ \\
\hline$O D C G_{\pi}^{S B}\left(v_{g}\right)$ & $\begin{array}{c}\text { demand } \\
d_{i}\end{array}$ & $\begin{array}{l}\text { choose a subset } \\
C \in\left\{a_{1}, \ldots, a_{i-1}\right\}\end{array}$ & $\left(C \cup\left\{a_{i}\right\}\right.$ ordered at random $)$ & $\begin{array}{l}\forall a_{k} \in C: p_{k}=d_{k} \\
p_{i}=v_{g}\left(\tilde{\pi}\left(C \cup\left\{a_{i}\right\}\right)\right)-\sum_{a_{k} \in C} d_{k} \\
\forall a_{k} \in N \backslash\left\{C \cup\left\{a_{i}\right\}\right\}: p_{k}=v_{g}\left(\left(a_{k}\right)\right)\end{array}$ \\
\hline
\end{tabular}

In Step 1 of all three mechanisms, a permutation of players $\pi$ is chosen randomly to determine the order of moves (the $i$ th player in $\pi$ makes the $i$ th move). Without loss of generality, the table assumes $\pi=\left(a_{1}, a_{2}, \ldots, a_{n}\right)$. In Step 2, the move of $a_{i}$ is to make a choice between (1) demanding $d_{i}$ in return for joining any coalition requested by a subsequent player, and (2) forming a coalition with (some of the previous players and ending the game. The main difference is in the way the mechanisms impose an order on the resulting multiplayer coalition.

\subsection{The $O D C G^{S B}\left(v_{g}\right)$ Mechanism and the $\sigma_{\pi, i}^{S B}$ Strategy}

The $O D C G^{S B}\left(v_{g}\right)$ mechanism is identical to $O D C G^{N R}\left(v_{g}\right)$ except for the following difference. In Option 2, the payoff of player $a_{i}$ in Eq. (14) becomes:

$$
v_{g}\left(\tilde{\pi}\left(C \cup\left\{a_{i}\right\}\right)\right)-\sum_{a_{k} \in C} d_{k}
$$

where $\tilde{\pi}\left(C \cup\left\{a_{i}\right\}\right)$ is an ordered coalition chosen uniformly at random from the set $\Pi^{C \cup\left\{a_{i}\right\}}$. This means $a_{i}$ can choose the identities (but not the order) of the agents who will join him in the same coalition. The order will be chosen randomly by the mechanism, only after the members are chosen by $a_{i}$.

Table I summarizes the differences between the SDCG mechanism proposed by Dasgupta and Chiu [1998] and the $O D C G^{N R}$ and $O D C G^{S B}$ mechanisms proposed in this article, where $p_{k}$ denotes the payoff of $a_{k}$.

\subsection{Properties of the Mechanisms}

Our key results with regards to the $O D C G^{N R}\left(v_{g}\right)$ and $O D C G^{S B}\left(v_{g}\right)$ mechanisms are presented in the following theorem.

THEOREM 4.1. Every SPNE of $O D C G^{N R}\left(v_{g}\right)$ and $O D C G^{S B}\left(v_{g}\right)$, where $v_{g}$ is strictly convex, has payoffs equal to the NR value, and the $S B$ value, respectively.

Proof. We start by recalling Eqs. (4), (8), and (13) in which either the Shapley value, the NR value or the SB value, respectively, are presented as the expected marginal contribution of player $a_{i}$ in permutation $\pi$, where $\pi \in \Pi(N)$ is selected uniformly at random. While this general functional form is the same for all three values, their differences stem:

-From the Different Underlying Value Function. In the case of the Shapley value this is the characteristic function $v$, whereas in the case of the NR value this is the generalized characteristic function $v_{g}$, and in the case of the SB value this is the average characteristic function for $v_{g}$, that is, it is $\bar{v}_{g}$; and 
-From the Different Definition of the Marginal Contribution. In the case of the Shapley value and the SB value, we have $\Delta_{v}\left(C_{\overleftarrow{\pi_{i}}}, a_{i}\right)$ and $\Delta_{\bar{v}_{g}}\left(C_{\overleftarrow{\pi}_{i}}, a_{i}\right)$, respectively (Eq. (5) for the value function $v$ and $\bar{v}_{g}$ ), whereas, for the NR value, we have $\Delta_{v_{g}}^{N R}\left(T, a_{i}\right)$ (Eq. (7)).

Step 1 of both our mechanisms is the same as Step 1 of the mechanism by Dasgupta and Chiu [1998]: a permutation $\pi$ is chosen uniformly at random. Therefore, what we need to show for each value is that our refinements account for the differences in the value function and the marginal contribution as outlined previously. To this end, let us consider the following lemmas:

Lemma 4.1.1. Given $\left(N, v_{g}\right)$, let $\pi \in \Pi(N)$ be an order of players chosen uniformly at random in Step 1 of $O D C G^{N R}\left(v_{g}\right)\left(O D C G^{S B}\left(v_{g}\right)\right)$. Then, for every player $a_{i} \in N$, Step 2 of the mechanism, that is, $O D C G_{\pi}^{N R}\left(v_{g}\right)\left(O D C G_{\pi}^{N R}\left(v_{g}\right)\right)$, is strategically equivalent to $S D C G_{\pi}\left(v_{g, \pi}\right)\left(S D C G_{\pi}\left(\bar{v}_{g}\right)\right)$.

Proof. We consider $O D C G^{N R}\left(v_{g}\right)$ first. By rules of this mechanism (see Table I), any coalition $C \cup\left\{a_{i}\right\}$ chosen by $a_{i}$ in Option 2 will be created as an ordered coalition $\operatorname{inv}\left(\pi\left(C \cup\left\{a_{i}\right\}\right)\right)$. Thus, the choices offered to $a_{i}$ by $O D C G^{N R}\left(v_{g}\right)$, that is, every $\operatorname{inv}(\pi(C \cup$ $\left.\left\{a_{i}\right\}\right)$ ) in the generalized characteristic function game $v_{g}$, are in fact equivalent to the choices offered to $a_{i}$ by $S D C G\left(v_{g, i n v(\pi)}\right)$, that is, every $C \cup\left\{a_{i}\right\}$ in the characteristic function game $v_{g, i n v(\pi)}$. This shows that Step 2 of $O D C G^{N R}\left(v_{g}\right)$ is strategically equivalent to Step 2 of $S D C G\left(v_{g, i n v(\pi)}\right)$.

Turning now to $O D C G^{S B}\left(v_{g}\right)$, by rules of this mechanism (see again Table I), any coalition $C \cup\left\{a_{i}\right\}$ chosen by $a_{i}$ in Option 2 will be created as an ordered coalition $\left(a_{i}, \tilde{\pi}(C)\right)$. Recall that $\tilde{\pi}(C)$ denotes a randomly ordered coalition made of players in $C$. Since player $a_{i}$ has to select $C$ without knowing how it will be ordered, his rational behavior is to consider the expected value of $C \cup\left\{a_{i}\right\}$ over all possible orders of $C$, bearing in mind that he will be placed in the first position of the ordered coalition (as per the rules of the mechanism). This expected value is

$$
\frac{1}{|C| !} \sum_{\tilde{\pi} \in \Pi(C)} v_{g}\left(\tilde{\pi}\left(C \cup\left\{a_{i}\right\}\right)\right),
$$

which is precisely $\bar{v}_{g}\left(C \cup\left\{a_{i}\right\}\right)$ (see Section 3). Thus, the choices offered to $a_{i}$ by ${ }_{O D C G}{ }^{S B}\left(v_{g}\right)$, that is, every $\left(a_{i}, \tilde{\pi}(C)\right)$ in the generalized characteristic function game $v_{g}$, are equivalent to the choices offered to $a_{i}$ by $S D C G\left(\bar{v}_{g}\right)$, that is, every $C \cup\left\{a_{i}\right\}$ in the characteristic function game $\bar{v}_{g}$. This shows that Step 2 of $O D C G^{S B}\left(v_{g}\right)$ is strategically equivalent to Step 2 of $S D C G\left(\bar{v}_{g}\right)$.

LEMMA 4.1.2. Given a (strictly) convex ordered game $\left(N, v_{g}\right)$, and a permutation $\pi \in$ $\Pi(N)$, the game $\left(N, v_{g, \pi}\right)$ is (strictly) convex.

Proof. We need to show that:

$$
v_{g, \pi}\left(C \cup\left\{a_{i}\right\}\right)-v_{g, \pi}(C)(>) \geq v_{g, \pi}\left(D \cup\left\{a_{i}\right\}\right)-v_{g, \pi}(D),
$$

where $a_{i} \in N \backslash C$ and $D \subset C \subset N$.

To this end, observe that every member of $D$ appears in $C$, and if a player, $a_{i}$, appears before another, $a_{j}$, in $\pi(D)$, then it will also appear before it in $\pi(C)$, as both coalitions are ordered according to $\pi$. Therefore, based on Definition 3.1, we have: $\pi(D) \vec{\subset} \pi(C)$. By a similar reasoning, we have $\pi\left(C \cup\left\{a_{i}\right\}\right) \vec{\subset} \pi\left(D \cup\left\{a_{i}\right\}\right)$. This, as well as the fact that $v_{g}$ is convex, implies the following (based on Definition 3.3):

$$
v_{g}\left(\pi\left(C \cup\left\{a_{i}\right\}\right)\right)-v_{g}(\pi(C))(>) \geq v_{g}\left(\pi\left(D \cup\left\{a_{i}\right\}\right)\right)-v_{g}(\pi(D)) .
$$


Moreover, by definition, we have $v_{g, \pi}(C)=v_{g}(\pi(C))$ for every $C \subseteq N$. This, together with Eq. (16), imply that Eq. (15) holds.

LEMMA 4.1.3. Given a (strictly) convex ordered game $\left(N, v_{g}\right)$, and a permutation $\pi \in$ $\Pi(N)$, the game $\left(N, \bar{v}_{g}\right)$ is (strictly) convex.

Proof. We know from Lemma 4.1.2 that, for all $\pi \in \Pi(N)$ and all $a_{i} \in N$, we have:

$$
v_{g, \pi}\left(C \cup\left\{a_{i}\right\}\right)-v_{g, \pi}(C)(>) \geq v_{g, \pi}\left(D \cup\left\{a_{i}\right\}\right)-v_{g, \pi}(D),
$$

for every $D \subset C \subset N \backslash\left\{a_{i}\right\}$. Thus:

$$
\frac{1}{|N| !} \sum_{\pi \in \Pi(N)}\left(v_{g, \pi}\left(C \cup\left\{a_{i}\right\}\right)-v_{g, \pi}(C)\right)(>) \geq \frac{1}{|N| !} \sum_{\pi \in \Pi(N)}\left(v_{g, \pi}\left(D \cup\left\{a_{i}\right\}\right)-v_{g, \pi}(D)\right) .
$$

Now observe that, for every coalition $C \subseteq N$, we have

$$
\frac{1}{|N| !} \sum_{\pi \in \Pi(N)}\left(v_{\pi}(C)\right)=\frac{1}{|C| !} \sum_{\pi \in \Pi(C)}\left(v_{\pi}(C)\right)=\bar{v}_{g}(C) .
$$

Equations (17) and (18) mean that

$$
\bar{v}_{g}\left(C \cup\left\{a_{i}\right\}\right)-\bar{v}_{g}(C)(>) \geq \bar{v}_{g}\left(D \cup\left\{a_{i}\right\}\right)-\bar{v}_{g}(D) .
$$

Next, building upon the above lemmas, as well as the result of Dasgupta and Chiu [1998], we prove the correctness of Theorem 4.1.

Dasgupta and Chiu [1998] showed that, for a convex characteristic function game $v$, all the SPNE of their mechanism, $S D C G(v)$, result in the Shapley value in expectation. They also showed that in the equilibrium any player $\alpha_{i}$ in the randomly chosen order $\pi$ (except for the last player) makes a demand that equals his contribution to the coalition consisting of all subsequent players in $\pi$ (we will denote this coalition by $\left.C_{\vec{\pi}_{i}}\right)$. For instance, given $\left(\left\{a_{1}, a_{2}, a_{3}\right\}, v\right)$ and $\pi=\left(a_{2}, a_{1}, a_{3}\right)$, player $a_{2}$ demands $d_{2}=$ $\Delta_{v}\left(C_{\vec{\pi}_{2}}, a_{2}\right)=v\left(\left\{a_{1}, a_{3}\right\} \cup\left\{a_{2}\right\}\right)-v\left(\left\{a_{1}, a_{3}\right\}\right)$, and player $a_{1}$ demands $d_{1}=\Delta_{v}\left(C_{\vec{\pi}_{1}}, a_{1}\right)=$ $v\left(\left\{a_{3}\right\} \cup\left\{a_{1}\right\}\right)-v\left(\left\{a_{3}\right\}\right)$. The last player in $\pi$, that is, $a_{3}$, forms the grand coalition, $\left\{a_{1}, a_{2}, a_{3}\right\}$, and satisfies the demands of $a_{1}$ and $a_{2}$, leaving him with a payoff equal to his marginal contribution to the empty set. That is, $a_{3}$ receives:

$$
v\left(\left\{a_{1}, a_{2}, a_{3}\right\}\right)-d_{2}-d_{1}=v\left(\left\{a_{3}\right\}\right),
$$

or, equivalently, $\Delta_{v}\left(C_{\vec{\pi}_{3}}, a_{3}\right)=v\left(\emptyset \cup\left\{a_{3}\right\}\right)-v(\emptyset)$.

Then, since the following holds:

$$
\mathbb{E}\left[\Delta_{C_{\vec{\pi}_{i}}, i}\right]=\frac{1}{|N| !} \sum_{\pi \in \Pi(N)} \Delta_{v}\left(C_{\vec{\pi}_{i}}, a_{i}\right)=\frac{1}{|N| !} \sum_{\pi \in \Pi(N)} \Delta_{v}\left(C_{\pi_{i}}, a_{i}\right) m=\mathbb{E}\left[\Delta_{C_{\overleftarrow{\pi_{i}}}, i}\right],
$$

the $S D C G(v)$ mechanism implements the Shapley value in expectation (see Eq. (4)).

From this result of Dasgupta and Chiu, as well as Lemmas 4.1.1 and 4.1.2, it follows that, for a convex generalized characteristic function, $v_{g}$, all SPNE of our mechanism $O D C G^{N R}\left(v_{g}\right)$ result in expectation in the following value:

$$
\phi_{i}^{*}\left(N, v_{g}\right)=\frac{1}{|N| !} \sum_{\pi \in \Pi(N)} \Delta_{v_{g, i n v(\pi)}}\left(C_{\vec{\pi}_{i}}, a_{i}\right)
$$

while all SPNE of our mechanism $O D C G^{S B}\left(v_{g}\right)$ result in expectation in

$$
\phi_{i}^{* *}\left(N, v_{g}\right)=\frac{1}{|N| !} \sum_{\pi \in \Pi(N)} \Delta_{\bar{v}_{g}}\left(C_{\vec{\pi}_{i}}, a_{i}\right)
$$


Equations (20) and (21) imply that, in order to prove Theorem 4.1, it suffices to prove that, the following two equations hold for all $a_{i} \in N$ :

$$
\phi_{i}^{*}\left(N, v_{g}\right)=\frac{1}{|N| !} \sum_{\pi \in \Pi(N)} \Delta_{v_{g, i n v(\pi)}}\left(C_{\vec{\pi}_{i}}, a_{i}\right)=\frac{1}{|N| !} \sum_{T \in \Pi(N)} \Delta_{v_{g}}^{N R}\left(T\left(a_{i}\right), a_{i}\right)=\phi_{i}^{N R}\left(N, v_{g}\right),
$$

and

$$
\phi_{i}^{* *}\left(N, v_{g}\right)=\frac{1}{|N| !} \sum_{\pi \in \Pi(N)} \Delta_{\bar{v}_{g}}\left(C_{\vec{\pi}_{i}}, a_{i}\right)=\frac{1}{|N| !} \sum_{T \in \Pi(N)} \Delta_{\bar{v}_{g}}\left(C_{\overleftarrow{\pi}_{i}}, a_{i}\right)=\phi_{i}^{S B}\left(N, v_{g}\right)
$$

Since the correctness of Eq. (23) is implied by Eq. (19), it remains to prove the correctness of Eq. (22). We will do it with the following lemma.

Lemma 4.1.4. Given $\left(N, v_{g}\right)$ and $a_{i} \in N$, there exists a bijection $f_{i}: \Pi(N) \rightarrow \Pi(N)$ such that for all $\pi \in N$ :

$$
\Delta_{v_{g, i n v(\pi)}}\left(C_{\vec{\pi}_{i}}, a_{i}\right)=\Delta_{v_{g}}^{N R}(\underbrace{f_{i}(\pi)\left(a_{i}\right)}_{=T\left(a_{i}\right)}, a_{i}) .
$$

PRoof. Let $f_{i}$ be defined as follows: for every $\pi=\left(a_{k_{1}}, \ldots, a_{k_{i-1}}, a_{k_{i}}, a_{k_{i+1}}, \ldots, a_{k_{n}}\right) \in$ $\Pi(N)$, where $a_{k_{i}}=a_{i}$, we have $f_{i}(\pi)=\operatorname{inv}(\pi)=\left(a_{k_{n}}, \ldots, a_{k_{i+1}}, a_{k_{i}}, a_{k_{i-1}}, \ldots, a_{k_{1}}\right)$. For this bijection, the right-hand side of Eq. (24) becomes:

$$
\Delta_{v_{g}}^{N R}\left(f_{i}(\pi)\left(a_{i}\right), a_{i}\right)=\Delta_{v_{g}}^{N R}\left(i n v(\pi)\left(a_{i}\right), a_{i}\right)=v_{g}\left(\left(a_{k_{n}}, \ldots, a_{k_{i-1}}, a_{k_{i}}\right)\right)-v_{g}\left(\left(a_{k_{n}}, \ldots, a_{k_{i-1}}\right)\right) .
$$

As for the left-hand side of Eq. (24), we have

$$
\begin{aligned}
\Delta_{v_{g, i n v(\pi)}}\left(C_{\vec{\pi}_{i}}, a_{i}\right) & =v_{g, i n v(\pi)}\left(C_{\vec{\pi}_{i}} \cup\left\{a_{i}\right\}\right)-v_{g, i n v(\pi)}\left(C_{\vec{\pi}_{i}}\right) \\
& =v_{g, i n v(\pi)}\left(\left\{a_{k_{i}}, a_{k_{i+1}}, \ldots, a_{n}\right\}\right)-v_{g, i n v(\pi)}\left(\left\{a_{k_{i+1}}, \ldots, a_{k_{n}}\right\}\right) .
\end{aligned}
$$

Since, by definition, we have $v_{g, \pi}(C)=v_{g}(\pi(C))$ for all $C \subseteq N$, we can rewrite Eq. (25) as follows:

$$
\begin{aligned}
\Delta_{v_{g, i n v(\pi)}}\left(C_{\vec{\pi}_{i}}, a_{i}\right) & =v_{g}\left(\operatorname{inv}(\pi)\left(\left\{a_{k_{i}}, a_{k_{i+1}}, \ldots, a_{n}\right\}\right)-v_{g}\left(\operatorname{inv}(\pi)\left(\left\{a_{k_{i+1}}, \ldots, a_{k_{n}}\right\}\right)\right)\right. \\
& =v_{g}\left(\left(a_{k_{n}}, \ldots, a_{k_{i-1}}, a_{k_{i}}\right)\right)-v_{g}\left(\left(a_{k_{n}}, \ldots, a_{k_{i-1}}\right)\right) .
\end{aligned}
$$

Hence, Eq. (24) holds.

This concludes the proof of Theorem 4.1.

In the next section, we will discuss equilibrium strategies.

\subsection{Strategies in the $O D C G^{N R}\left(v_{g}\right)$ and $O D C G^{S B}\left(v_{g}\right)$ Mechanisms}

In section, we present an equilibrium strategy of player $a_{i}$. It has two versions; one for $O D C G^{N R}\left(v_{g}\right)$, denoted by $\sigma_{\pi, i}^{N R}$, and the other for $O D C G^{S B}\left(v_{g}\right)$, denoted by $\sigma_{\pi, i}^{S B}$. Before we introduce these strategies, we need to introduce additional notation. Let $H_{i}=\mathbb{R}^{i-1}$ be the set of all possible histories that $a_{i}$ may face; every history in $H_{i}$ represents a unique set of demands $\left(d_{1}, \ldots, d_{i-1}\right)$. Now, let $a_{i}$ face the history $h=\left(d_{1}, \ldots, d_{i-1}\right)$ and let $D$ be any subset of players from $\left\{a_{i}, a_{i+1}, \ldots, a_{n}\right\}$. We define $M_{h, \pi}^{N R}(D)$ as follows:

$$
M_{h, \pi}^{N R}(D)=\max _{C \subseteq\left\{a_{1}, \ldots, a_{i-1}\right\}}\left\{v_{g}(i n v(\pi(D \cup C)))-\sum_{a_{j} \in C} d_{j}\right\} .
$$

That is, $M_{h, \pi}^{N R}(D)$ is the maximum payoff that coalition $D$ can obtain for itself if it is allowed to choose a set of new members, denoted by $C$, from the players 
that precede $a_{i}$, bearing in mind that the mechanism will enforce the formation of $\operatorname{inv}(\pi(D \cup C))$. Furthermore, for every $j \in\{i, \ldots, n\}$, let $\mathcal{P}_{\pi}^{h}(i, j)$ denote the following linear program:

$\mathcal{P}_{\pi}^{h}(i, j): \max _{d_{i}, \ldots, d_{j}} d_{i} \quad$ subject to:

$$
\begin{aligned}
& d_{k_{1}}+\cdots+d_{k_{m}} \geq M_{h, \pi}^{N R}\left(\left\{a_{k_{1}}, \ldots, a_{k_{m}}\right\}\right) \text { for all } k_{1}, \ldots, k_{m} \text { where } i \leq k_{1}<\cdots<k_{m} \leq j \\
& \text { and } d_{i}+\cdots+d_{j}=M_{h, \pi}^{N R}\left(\left\{a_{i}, \ldots, a_{j}\right\}\right) .
\end{aligned}
$$

In other words, $\mathcal{P}_{\pi}^{h}(i, j)$ computes for players $a_{i}, \ldots, a_{j}$ and characteristic function $M_{h, \pi}^{N R}($.$) a core allocation that gives a_{i}$ the largest payoff. Next, we outline the conditions that characterize the maximal program for player $a_{i}$.

Definition 4.2. Given a history $h=\left(d_{1}, \ldots, d_{i-1}\right)$, a program $\mathcal{P}_{\pi}^{h}(i, j)$ for player $a_{i}$ is called a maximal program if

一there exists a solution $\left(d_{i}, \ldots, d_{j}\right)$ for $\mathcal{P}_{\pi}^{h}(i, j)$, that is, the program is feasible. ${ }^{4}$

一no other feasible program $\mathcal{P}_{\pi}^{h}(i, k): k \neq j$ has a greater objective-function value;

-no other feasible program $\mathcal{P}_{\pi}^{h}(i, k): k>j$ has the same objective-function value.

This definition implies that every maximal program is unique. We are now ready to introduce our strategy. Recall that every $a_{i}: 1 \leq i<n$ must choose between two options: either to specify a demand or select a subset of $\left\{a_{1}, \ldots, a_{i-1}\right\}$, while $a_{n}$ has only one option, which is to select a subset from $\left.\left\{a_{1}, \ldots, a_{n-1}\right\}\right)$. This implies that a strategy of $a_{i}: 1 \leq i<n$ is a mapping from $H_{i}$ to $\mathbb{R} \cup 2^{\left\{a_{1}, \ldots, a_{i-1}\right\}}$, while a strategy of $a_{n}$ is a mapping from $H_{n}$ to $2^{\left\{a_{1}, \ldots, a_{n-1}\right\}}$. Our strategy, $\sigma_{\pi, i}^{N R}$, proceeds as follows:

-If $\mathcal{P}_{\pi}^{h}(i, j)$ is maximal, where $j>i$, then demand the value of the objective function in $\mathcal{P}_{\pi}^{h}(i, j)$

-If $\mathcal{P}_{\pi}^{h}(i, i)$ is maximal, then form the ordered coalition $\operatorname{inv}\left(\pi\left(C^{*} \cup\left\{a_{i}\right\}\right)\right)$, where $C^{*}$ solves:

$$
M_{h, \pi}^{N R}\left(\left\{a_{i}\right\}\right)=\max _{C \subseteq\left\{a_{1}, \ldots, a_{i-1}\right\}}\left\{v_{g}\left(\operatorname{inv}\left(\pi\left(C \cup\left\{a_{i}\right\}\right)\right)\right)-\sum_{a_{j} \in C} d_{j}\right\} .
$$

If there are more than one such argmaxes, then, following Dasgupta and Chiu [1998], we adopt the lexicographic tie-breaking rule.

As for the strategy $\sigma_{\pi, i}^{S B}$, it is identical to $\sigma_{\pi, i}^{N R}$ except for the following difference. Every $M_{h, \pi}^{N R}(D)$ is replaced with $M_{h, \pi}^{S B}(D)$, which is defined as follows:

$$
M_{h, \pi}^{S B}(D)=\max _{C \subseteq\left\{a_{1}, \ldots, a_{i-1}\right\}}\left\{\frac{1}{|C| !} \sum_{T \in \Pi\left(C \cup\left\{a_{i}\right\}\right)} v_{g}(T)-\sum_{a_{j} \in C} d_{j}\right\} .
$$

\section{REPRESENTATION}

In this section, we consider the issue of efficient computation of the NR and SB values. Recall that a straightforward computation of the NR value, using either formulas (9) or (8), requires iterating over all ordered coalition. The same holds for the SB value. Now since, for any game of $n$ players, there are $\sum_{i=1}^{n}\left(\left(\begin{array}{l}n \\ i\end{array}\right) \times i\right.$ !) ordered coalitions,

${ }^{4}$ We note that the trivial program $\mathcal{P}_{\pi}^{h}(i, i)$ is always feasible. 
such an approach quickly becomes prohibitive with increasing $n .^{5}$ To tackle this computational problem, we introduce in this section a compact representation scheme for games with ordered coalitions and evaluate its properties. We take as our starting point the marginal contribution nets (MC-Nets) representation by Ieong and Shoham [2006] and its extension, read-once marginal contribution nets (or read-once MCNets) by Elkind et al. [2009]. This representation has a number of desirable properties: it is fully expressive, concise for many characteristic function games of interest, and facilitates a very efficient technique for computing the Shapley value. Our representation, which we call generalized read-once $M C$-Nets, also has these desirable properties.

This section is divided into four subsections. In Section 5.1, we introduce the generalized read-once $M C$-Nets representation to represent generalized characteristic function games compactly. After that, in Sections 5.2 and 5.3, we prove that this representation facilitates an efficient computation of the NR value and the SB value, respectively. Finally, in Section 5.4, we present a sample application of generalized read-once MC-Nets to compute game-theoretic centrality based on the NR and SB values.

\subsection{Generalized Read-Once MC-Nets}

Before presenting our representation (for generalized characteristic function games), we provide a brief description of MC-Nets (for characteristic function games). Specifically, with MC-Nets, a game is represented by a set of rules $\mathcal{R}$, where each rule is of the form Formula $\longrightarrow V$ in which Formula is a propositional formula over $N$, and $V$ is a real number. A coalition $C$ is said to meet a given formula $\mathcal{F}$ if and only if $\mathcal{F}$ evaluates to true when the values of all Boolean variables that correspond to players in $C$ are set to true, and the values of all Boolean variables that correspond to players not in $C$ are set to false. We write $C \models \mathcal{F}$ to mean that $C$ meets $\mathcal{F}$. In MC-Nets, if coalition $C$ does not meet any rule then its value is 0 . Otherwise, the value of $C$ is the sum of every $V$ from the rules of which the Formula are met by $C$. More formally:

$$
v(C)=\sum_{\mathcal{R} \ni \mathcal{F} \rightarrow V: C \models \mathcal{F}} V .
$$

Ieong and Shoham [2006] showed that, given an MC-Net representation in which rules are made only of conjunctions of positive and/or negative literals, the Shapley value can be computed in time linear in the number of rules. Similarly to Elkind et al. [2009], we will call such rules "basic rules", and the representation based on them basic MC-Nets. Any basic rule can be written as $z \wedge \neg z^{\prime} \rightarrow V$, where $z$ and $z^{\prime}$ are conjunctions of positive and negative literals, respectively. Now, taking advantage of the additivity property of the Shapley value, every basic rule can be considered separately as a game on its own. It can be shown that the Shapley values for any $a_{i}$ in $z$ and $a_{j}$ in $\neg z^{\prime}$ are

$$
\frac{V}{|z|\left(\begin{array}{c}
|z|+\left|\neg z^{\prime}\right| \\
\left|\neg z^{\prime}\right|
\end{array}\right)} \text { and } \frac{-V}{\left|\neg z^{\prime}\right|\left(\begin{array}{c}
|z|+\left|\neg z^{\prime}\right| \\
|z|
\end{array}\right)} \text {. }
$$

respectively, where $|z|$ and $\left|\neg z^{\prime}\right|$ denote the number of elements in $z$ and $\neg z^{\prime}$ respectively.

Elkind et al. [2009] defined a class of MC-Net rules, called read-once MC rules, which are considerably more succinct than basic rules, while enjoying similarly attractive

\footnotetext{
${ }^{5}$ For instance, the size of the generalized characteristic function for $n=5$ is 325 , whereas for $n=10$ is 9864100
} 
computational properties. The syntax of read-once Boolean formulas is as follows:

$$
\begin{array}{ll}
A:=a_{i} \mid \neg a_{i} & i \in\{1 \ldots n\}, \\
\mathcal{F}:=(\mathcal{F})|\mathcal{F} \wedge \mathcal{F}| \mathcal{F} \vee \mathcal{F}|\mathcal{F} \oplus \mathcal{F}| A, &
\end{array}
$$

where each literal $a_{i}$ can only appear once. In other words, this formula is a binary rooted tree with positive or negative literals on leafs and internal nodes labeled with one of the following logical connectives: conjunction $(\wedge)$, disjunction $(\vee)$ and exclusive disjunction $(\oplus)$.

Elkind et al. [2009] proved that, in general MC-Nets, where each literal can appear in a formula many times, the problem of computing the Shapley value is intractable (i.e., NP-hard). However, for the read-once Boolean formulas, the authors presented a polynomial-time algorithm to compute the Shapley value.

Having presented the MC-Net representation for characteristic function games, we now present our extension of MC-Nets to generalized characteristic function games. More specifically, we extend the rules so that the Formula on the left-hand side of a rule is applicable to an ordered coalition. We introduce two atomic formulas:

$$
\begin{aligned}
\text { basic atomic formula (BAF) } & \left\{a_{m}, \ldots, a_{n}\right\}, \\
\text { ordered atomic formula (OAF) } & \left\langle a_{i}, \ldots, a_{j}\right\rangle .
\end{aligned}
$$

The ordered coalition $T$ meets a basic atomic formula, denoted $\mathcal{F}_{B A F}$, if all literals occurring in $\mathcal{F}_{B A F}$ belong to $T$. More formally

$$
T \models \mathcal{F}_{B A F} \Longleftrightarrow \forall a_{i} \in \mathcal{F}_{B A F}, a_{i} \in T .
$$

Example 5.1. Let $N=\left\{a_{1}, \ldots, a_{10}\right\}$ and consider $T=\left(a_{5}, a_{1}, a_{4}, a_{3}, a_{2}\right)$. We have

$$
\begin{aligned}
& T \vDash\left\{a_{2}, a_{5}\right\}, \\
& T \not \models\left\{a_{5}, a_{2}, a_{4}, a_{6}\right\} .
\end{aligned}
$$

Now, we introduce an ordered atomic formula, denoted $\mathcal{F}_{O A F}$. This formula is our crucial extension to MC-Nets; it allows us to fully express the values of all ordered coalitions. Specifically, the ordered coalition $T$ meets $\mathcal{F}_{O A F}$ if all literals occurring in $\mathcal{F}_{O A F}$ appear in $T$ in the same order as in the $\mathcal{F}_{O A F}$ formula.

To state this more formally, let us first introduce the notion of an ordered coalition corresponding to $\mathcal{F}_{O A F}$. To this end, for every $\mathcal{F}_{O A F}=\left\langle a_{i}, \ldots, a_{j}\right\rangle$, the corresponding ordered coalition of $\mathcal{F}_{O A F}$ is simply $S^{O A F}=\left(a_{i}, \ldots, a_{j}\right)$. The main difference between the two is that $a_{i}$ is a literal in $\mathcal{F}_{O A F}$, while it is an agent in $S^{O A F}$. Now, given a particular $\mathcal{F}_{O A F}$ and an ordered coalition $S^{O A F}$ corresponding to it, the following holds for every $T \in \mathcal{T}$ :

$$
T \models \mathcal{F}_{O A F} \Longleftrightarrow S^{O A F} \underline{\subseteq} T .
$$

In other words, $S^{O A F}$ is a subsequence of $T$. This has a natural interpretation: when a group of players joins any coalition in a certain order they contribute to it a certain value. Consider the following example.

Example 5.2. Let $N=\left\{a_{1}, \ldots, a_{10}\right\}$ and consider $T=\left(a_{5}, a_{1}, a_{4}, a_{3}, a_{2}\right)$. We have:

$$
\begin{aligned}
& T \vDash\left\langle a_{5}, a_{3}, a_{2}\right\rangle, \\
& T \not \models\left\langle a_{5}, a_{2}, a_{3}\right\rangle, \\
& T \not \models\left\langle a_{5}, a_{3}, a_{2}, a_{6}\right\rangle .
\end{aligned}
$$


Now, we are able to formalize our representation which we call the generalized readonce MC-Nets. Specifically, in this representation, a rule, called generalized read-once rule, is as follows:

$$
\mathcal{F} \rightarrow V
$$

where $V$ is a real value and the syntax of the generalized formula $\mathcal{F}$ is:

$$
\begin{aligned}
& A:=\mathcal{F}_{B A F} \mid \mathcal{F}_{O A F}, \\
& \mathcal{F}:=(\mathcal{F})|\neg \mathcal{F}| \mathcal{F} \wedge \mathcal{F}|\mathcal{F} \vee \mathcal{F}| \mathcal{F} \oplus \mathcal{F} \mid A,
\end{aligned}
$$

and each literal can only appear once in the formula. This generalized formula is a binary rooted tree with atomic formulas on leafs and internal nodes labeled with one of the following logical connectives: conjunction $(\wedge)$, disjunction $(\vee)$ or exclusive disjunction $(\oplus)$. The logical connectives have the following standard interpretation:

$$
\begin{aligned}
& T \vDash \neg \mathcal{F} \quad \Longleftrightarrow T \not \models \mathcal{F}, \\
& T \vDash \mathcal{F}_{1} \wedge \mathcal{F}_{2} \Longleftrightarrow T \vDash \mathcal{F}_{1} \text { and } T \vDash \mathcal{F}_{2} \text {, } \\
& T \vDash \mathcal{F}_{1} \vee \mathcal{F}_{2} \Longleftrightarrow T \vDash \mathcal{F}_{1} \text { or } T \vDash \mathcal{F}_{2} \text {, } \\
& T \vDash \mathcal{F}_{1} \oplus \mathcal{F}_{2} \Longleftrightarrow T \vDash\left(\mathcal{F}_{1} \vee \mathcal{F}_{2}\right) \wedge \neg\left(\mathcal{F}_{1} \wedge \mathcal{F}_{2}\right) .
\end{aligned}
$$

Example 5.3. Let $N=\left\{a_{1}, \ldots, a_{10}\right\}$ and consider $T=\left(a_{5}, a_{1}, a_{4}, a_{3}, a_{2}\right)$. We have

$$
\begin{aligned}
& T \vDash\left(\left\{a_{2}, a_{5}\right\} \wedge\left\langle a_{1}, a_{3}\right\rangle\right) \vee\left\{a_{4}, a_{7}, a_{9}\right\}, \\
& T \not \models\left\langle a_{4}, a_{5}, a_{3}\right\rangle \oplus\left(\left\{a_{1}\right\} \wedge \neg\left\{a_{2}\right\}\right) .
\end{aligned}
$$

A generalized coalitional game $(N, v)$ can be represented by a tuple $(N, \mathcal{G R})$, where $\mathcal{G R}$ is a finite set of generalized rules. The value $v_{g}(T)$ of an ordered coalition $T$ is defined as the sum of all $V$ from the generalized read-once rules that are met by $T$. More formally:

$$
v_{g}(T)=\sum_{\mathcal{G} \mathcal{R} \ni \mathcal{F} \rightarrow V: T \models \mathcal{F}} V
$$

The following example presents a simple application of generalized read-once MC-Nets to the scheduling problem.

Example 5.4. Let $G=(V, E)$ be a directed acyclic graph representing a task-based domain, where each vertex in $V=\left\{v_{1}, \ldots, v_{n}\right\}$ represents a task. Each edge $\left(v_{i}, v_{j}\right) \in E$ illustrates that task $v_{i}$ has to be completed before task $v_{j}$. We say that the job is done if all tasks of a given graph $G$ are completed, in which case we get some revenue. Next, we show how each such graph can be represented by a single generalized read-once rule, where agents represent tasks. In particular, each formula in the rule represents an edge in $G$, as follows:

$$
\bigwedge_{\left(a_{i}, a_{j}\right) \in E}\left\langle a_{i}, a_{j}\right\rangle \rightarrow \text { revenue of the job defined by } G \text {. }
$$

An ordered coalition $T$ represents the workforce that can finish tasks in a given order. The total revenue of $T$ is then equal to the sum of revenues of all jobs feasible by $T$.

Having defined our representation formally, we now evaluate its properties. We start with expressiveness. 
Proposition 5.5 (Expressiveness). Every ordered coalitional game can be expressed using generalized read-once MC-Nets.

PRoOF. For any arbitrary generalized characteristic function $v_{g}$, we show how to construct a set of rules $\mathcal{G} \mathcal{R}$ such that Eq. (32) holds for every $T \in \mathcal{T}$.

We will use only OAF to define a recursive set starting from singleton sequences and empty set $\mathcal{G R}_{0}=\emptyset$. Let $S^{O A F}$ be the ordered coalition corresponding to $\mathcal{F}_{O A F}$, and let $\left|\mathcal{F}_{O A F}\right|$ be the size of this formula.

$$
\begin{aligned}
& \mathcal{G} \mathcal{R}_{1}=\left\{\left\langle a_{i}\right\rangle \rightarrow v_{g}\left(\left(a_{i}\right)\right)\right\} \\
& \mathcal{G} \mathcal{R}_{n}=\left\{\mathcal{F}_{O A F} \rightarrow v_{g}\left(S^{O A F}\right)-\sum_{k=1}^{n-1} \sum_{\mathcal{G R}_{k} \ni \mathcal{F} \rightarrow V: S^{O A F} \models \mathcal{F}} V\right\} \forall\left|\mathcal{F}_{O A F}\right|=n .
\end{aligned}
$$

Finally, we get $\mathcal{G R}=\bigcup_{i} \mathcal{G} \mathcal{R}_{i}$.

In this proof of Proposition 5.5, it emerges that only OAFs are necessary in order to guarantee that the generalized MC-Nets are fully expressive. In the following basic example, we present that in some games the BAF gives us desirable succinctness.

Example 5.6. Let $N=\left\{a_{1}, \ldots, a_{n}\right\}$ be the set of tasks. In order to get profit $V$, all tasks must be done in arbitrary chosen order. It is clear that representing this problem using only OAF requires $n$ ! number of formulas:

$$
\begin{aligned}
\left\langle a_{1}, \ldots, a_{n}\right\rangle & \rightarrow V, \\
\vdots & \\
\left\langle a_{n}, \ldots, a_{1}\right\rangle & \rightarrow V .
\end{aligned}
$$

By introducing BAF, we can get exponentially more concise notation: $\left\{a_{1}, \ldots, a_{n}\right\} \rightarrow V$.

It is worth making some observations about the conciseness of our representation scheme. Our rule-based representation scheme is ultimately based on propositional formulae, and the use of these formulae is ultimately to define a Boolean function. The use of propositional formulae to define Boolean functions is very standard: a very fundamental result in the theory of propositional logic is that any Boolean function of $n$ Boolean arguments can be represented by a propositional formula in which the variables correspond to these arguments. For many Boolean functions, it might be possible to obtain compact propositional formulae to represent them, but crucially, this will not always be possible; some Boolean functions will require formulae of size exponential in the number of variables (see, e.g., Boppana and Sipser [1990, p. 763]). It is, in fact, trivial to produce examples of characteristic functions whose representation in our framework is exponentially more succinct than explicitly listing the value of every coalition, but from this discussion, it also follows that our representation cannot guarantee compactness in all cases. Finally, note that in the worst case, we can represent a characteristic function with one rule for every possible input, which essentially corresponds to the idea of listing the value of every coalition. Thus, in the worst case, our representation scheme is no worse than simply listing the value of every input. More formally:

Corollary 5.7 (Conciseness). Compared to the generalized function game representation, generalized read-once MC-nets are at least as concise, and for certain games exponentially more compact. 


\subsection{Computing the NR Value with Generalized Read-Once MC-Nets}

In this section, we discuss the computational properties of the generalized read-once MC-Net representation with respect to calculating the Nowak-Radzik value (i.e., the $\mathrm{NR}$ value). We start our computational analysis with some observations about atomic formulas. First, observe that all players occurring in some $\mathcal{F}_{B A F}$ are indistinguishable. Furthermore, taking into account that each player can appear in at most one formula, $\phi^{N R}$ will assign to these players the same values. The case of $\mathcal{F}_{O A F}$ is different. Here, the NR value for all players except the last one is zero (see Example 3.4).

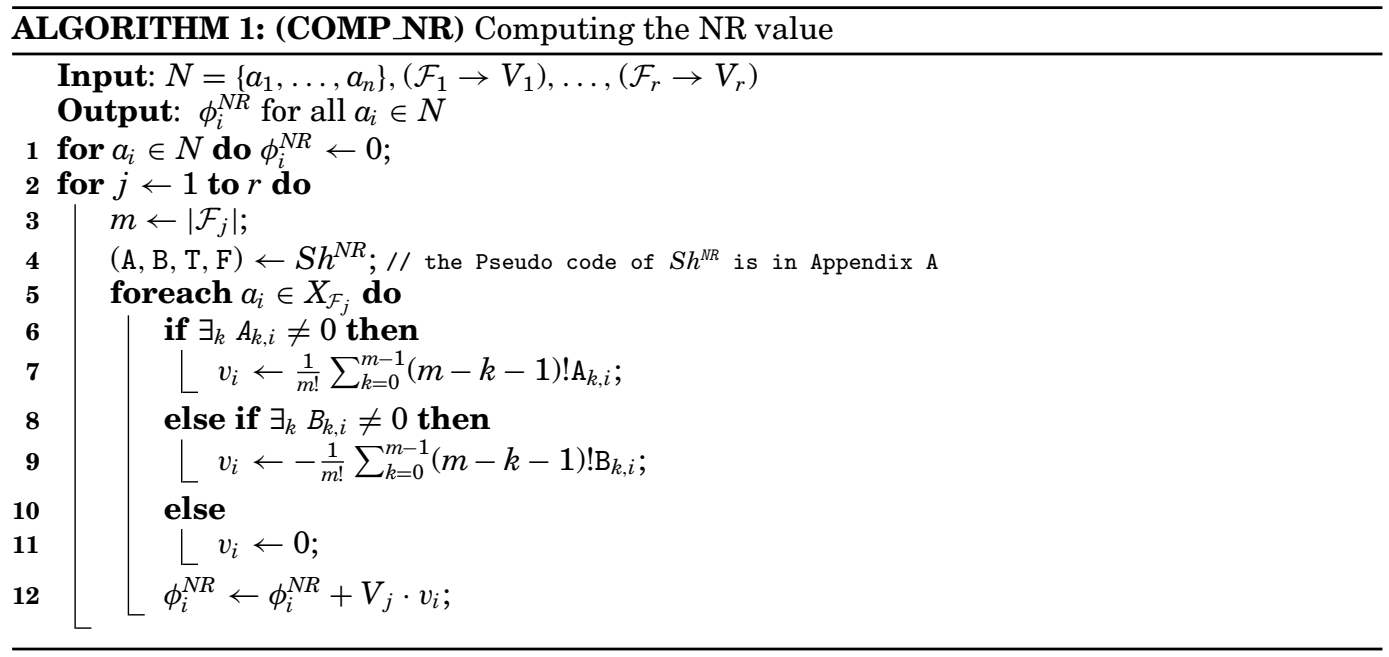

TheOREM 5.8. Given a generalized coalitional game $(N, \mathcal{G R})$, Algorithm 1 computes the NR value ( $\phi_{i}^{N R}$ for all $\left.a_{i} \in N\right)$ in polynomial time.

Proof. We start by introducing necessary notation, some of which is in the pseudocode of Algorithm 1 . For each formula $\mathcal{F}$, we denote by $X_{\mathcal{F}}$ the sets of players occurring in $\mathcal{F}$, and by $|\mathcal{F}|$ the size of $X_{\mathcal{F}}$, and by $\mathcal{T}_{\mathcal{F}}$ the set of all ordered coalitions within the set $X_{\mathcal{F}}$, that is, $\mathcal{T}_{\mathcal{F}}=\bigcup_{C \subseteq X_{\mathcal{F}}} \Pi(C)$. For all $a_{i} \in X_{\mathcal{F}}$ and $k \in\{0, \ldots, n\}$, we define the following quantities:

$$
\begin{aligned}
& \left.\mathrm{A}_{k, i}(\mathcal{F})=\left|T \in \mathcal{T}_{\mathcal{F}}:\right| T \mid=k, a_{i} \notin T, T \not \models \mathcal{F},\left(T, a_{i}\right) \vDash \mathcal{F}\right) \mid, \\
& \left.\mathrm{B}_{k, i}(\mathcal{F})=\left|T \in \mathcal{T}_{\mathcal{F}}:\right| T \mid=k, a_{i} \notin T, T \vDash \mathcal{F},\left(T, a_{i}\right) \not \models \mathcal{F}\right) \mid, \\
& \mathrm{T}_{k}(\mathcal{F})=\left|T \in \mathcal{T}_{\mathcal{F}}:\right| T|=k, T \vDash \mathcal{F}|, \\
& \mathrm{F}_{k}(\mathcal{F})=\left|T \in \mathcal{T}_{\mathcal{F}}:\right| T|=k, T \not \models \mathcal{F}| .
\end{aligned}
$$

The first quantity $\mathrm{A}_{k, i}(\mathcal{F})$ is the number of ordered coalitions from $\mathcal{T}_{\mathcal{F}}$ of a given size $k$ (i.e., containing $k$ players) to which the addition of player $a_{i}$ at the end of the coalition causes the formula $\mathcal{F}$ to become satisfied. In contrast, the second quantity $\mathrm{B}_{k, i}$ is the number of coalitions to which adding player $a_{i}$ causes the formula $\mathcal{F}$ to become unsatisfied. The last two quantities, $\mathrm{T}_{k}(\mathcal{F})$ and $\mathrm{F}_{k}(\mathcal{F})$, count the number of ordered coalition being satisfied, and not satisfied, respectively, by the formula $\mathcal{F}$. These quantities can be computed recursively as follows. Let us first focus on the case where $\mathcal{F}$ is an atomic formula. This case can be divided further into two cases: 
$-\mathcal{F}=\left\{a_{1}, \ldots, a_{r}\right\}:$ Here, $\mathrm{A}_{k, i}=(r-1)$ ! if $k=r-1$ and $i \in X_{\mathcal{F}}$, otherwise $\mathrm{A}_{k, i}=0 . \mathrm{B}_{k, i}$ always equals 0 . $\mathrm{T}_{k}=r$ ! if $k=r$, otherwise $\mathrm{T}_{k}=0$. Finally, $\mathrm{F}_{k}=k$ ! if $k<r$, otherwise $\mathrm{F}_{k}=0$.

$-\mathcal{F}=\left\langle a_{1}, \ldots, a_{r}\right\rangle:$ Here $\mathrm{A}_{k, i}=1$ if $k=r-1$ and $i=r$, otherwise $\mathrm{A}_{k, i}=0 . \mathrm{B}_{k, i}$ always equals $0 . \mathrm{T}_{k}=1$ if $k=r$, otherwise $\mathrm{T}_{k}=0$. Finally, $\mathrm{F}_{k}=k !-\mathrm{T}_{k}$ if $k \leq r$, otherwise $\mathrm{F}_{k}=0$.

Having discussed the case where $\mathcal{F}$ is an atomic formula, we now consider the case where $\mathcal{F}$ contains logical connectives. If such a connective involves two subformulas $\mathcal{F}_{1}$ and $\mathcal{F}_{2}$, then we know that $a_{i}$ can appear in $\mathcal{F}_{1}$ or $\mathcal{F}_{2}$, but not in both. We also know that logical connectives used in the generalized read-once MC-Nets are symmetric, so we will consider only one case, $a_{i} \in \mathcal{F}_{1}$; the other is analogous.

In our analysis, for every two disjoint ordered coalitions, $T_{1}$ and $T_{2}$, we define a conflation of $T_{1}$ and $T_{2}$ as an ordered coalition $T$ such that (1) every agent in $T$ appears in $T_{1}$ or $T_{2}$, and (2) the order of the agents in $T_{1}$ is retained in $T$, and the order of those in $T_{2}$ is also retained in $T$. The set of every conflation of $T_{1}$ and $T_{2}$ will be denoted $T_{1} \times T_{2}$. More formally:

$$
T \in T_{1} \times T_{2} \Longleftrightarrow X_{T}=X_{T_{1}} \cup X_{T_{2}} \wedge T^{\prime} \vec{\subset} T \wedge T_{2} \vec{\subset} T,
$$

where $X_{T}$ denotes a set of players in $T$. We note that, if $T_{1} \vDash \mathcal{F}$ and $X_{\mathcal{F}} \cap T_{2}=\emptyset$, then $T \vDash \mathcal{F} \forall T \in\left(T_{1} \times T_{2}\right)$.

Proposition 5.9. Two ordered coalitions $T_{1}$ and $T_{2}$ can be conflated in $\left(\begin{array}{c}\left|T_{1}\right|+\left|T_{2}\right| \\ \left|T_{2}\right|\end{array}\right)$ ways.

Proof. Consider an sequence of $\left|T_{1}\right|+\left|T_{2}\right|$ empty slots, where each slot can take an agent. Out of all these slots, choose any $\left|T_{2}\right|$ slots, and place in them the agents from $T_{2}$ while retaining their order. As for the remaining slots, place in them the agents from $T_{1}$ while retaining their order. Clearly, every choice of the $\left|T_{2}\right|$ slots results in a unique conflation, and every conflation can be constructed by exactly one such choice of the $\left|T_{2}\right|$ slots. The number of conflations is then $\left(\begin{array}{c}\left|T_{1}\right|+1+\left|T_{2}\right|-1 \\ \left|T_{2}\right|\end{array}\right)$.

Next, we show how to compute $\mathrm{A}_{k, i}, \mathrm{~B}_{k, i}, \mathrm{~T}_{k}$ and $\mathrm{F}_{k}$. We will do this for each of the possible logical connective (i.e., $\neg, \wedge, \vee$ and $\oplus$ ) separately:

$-\mathcal{F}=\neg \mathcal{F}_{1}$. In this case, the negation swaps quantities as follows. For all $i$ and $k$, we have $\mathrm{A}_{k, i}(\mathcal{F})=\mathrm{B}_{k, i}\left(\mathcal{F}_{1}\right), \mathrm{B}_{k, i}(\mathcal{F})=\mathrm{A}_{k, i}\left(\mathcal{F}_{1}\right), \mathrm{T}_{k}(\mathcal{F})=\mathrm{F}_{k}\left(\mathcal{F}_{1}\right)$, and $\mathrm{F}_{k}(\mathcal{F})=\mathrm{T}_{k}\left(\mathcal{F}_{1}\right)$.

$-\mathcal{F}=\mathcal{F}_{1} \wedge \mathcal{F}_{2}$. In this case, let $T \in T_{1} \times T_{2}$, where $T_{1} \in \Pi\left(X_{\mathcal{F}_{1}}\right),\left|T_{1}\right|=s$ and $T_{2} \in$ $\Pi\left(X_{\mathcal{F}_{2}}\right),\left|T_{2}\right|=k-s$. We have $\left(T, a_{i}\right) \vDash \mathcal{F}$ if and only if $\left(T_{1}, a_{i}\right) \vDash \mathcal{F}_{1}$ and $T_{2} \vDash \mathcal{F}_{2}$. In this case, $T \not \models \mathcal{F}$ if and only if $T_{1} \not \models \mathcal{F}_{1}$. Consequently, using Proposition 5.9, we get:

$$
\mathrm{A}_{k, i}=\sum_{s=0}^{k}\left(\begin{array}{c}
k \\
k-s
\end{array}\right) \mathrm{A}_{s, i}\left(\mathcal{F}_{1}\right) \mathrm{T}_{k-s}\left(\mathcal{F}_{2}\right) .
$$

Furthermore, we have $T \vDash \mathcal{F}$ if and only if $T_{1} \vDash \mathcal{F}_{1}$ and $T_{2} \vDash \mathcal{F}_{2}$. Additionally in this case, we have: $\left(T, a_{i}\right) \not \models \mathcal{F} \Leftrightarrow\left(T_{1}, a_{i}\right) \not \models \mathcal{F}_{1}$. Consequently,

$$
\mathrm{B}_{k, i}=\sum_{s=0}^{k}\left(\begin{array}{c}
k \\
k-s
\end{array}\right) \mathrm{B}_{s, i}\left(\mathcal{F}_{1}\right) \mathrm{T}_{k-s}\left(\mathcal{F}_{2}\right) .
$$


Eventually, for $\mathrm{T}_{k}$ and $\mathrm{F}_{k}$, we have:

$$
\begin{aligned}
\mathrm{T}_{k} & =\sum_{s=0}^{k}\left(\begin{array}{c}
k \\
k-s
\end{array}\right) \mathrm{T}_{s}\left(\mathcal{F}_{1}\right) \mathrm{T}_{k-s}\left(\mathcal{F}_{2}\right), \\
\mathrm{F}_{k} & =\sum_{s=0}^{k}\left(\begin{array}{c}
k \\
k-s
\end{array}\right)\left(\mathrm{F}_{s}\left(\mathcal{F}_{1}\right) \mathrm{F}_{k-s}\left(\mathcal{F}_{2}\right)+\mathrm{F}_{s}\left(\mathcal{F}_{1}\right) \mathrm{T}_{k-s}\left(\mathcal{F}_{2}\right)+\mathrm{T}_{s}\left(\mathcal{F}_{1}\right) \mathrm{F}_{k-s}\left(\mathcal{F}_{2}\right)\right) .
\end{aligned}
$$

$-\mathcal{F}=\mathcal{F}_{1} \vee \mathcal{F}_{2}$. In this case, let $T \in T_{1} \times T_{2}$, where $T_{1} \in \Pi\left(X_{\mathcal{F}_{1}}\right),\left|T_{1}\right|=s$ and $T_{2} \in$ $\Pi\left(X_{\mathcal{F}_{2}}\right),\left|T_{2}\right|=k-s$. We know that $T \not \models \mathcal{F}$ if and only if $T_{1} \not \models \mathcal{F}_{1}$ and $T_{2} \not \models \mathcal{F}_{2}$. Furthermore, in this case, it holds that $\left(T, a_{i}\right) \vDash \mathcal{F} \Leftrightarrow\left(T_{1}, a_{i}\right) \vDash \mathcal{F}_{1}$. Thus,

$$
\mathrm{A}_{k, i}=\sum_{s=0}^{k}\left(\begin{array}{c}
k \\
k-s
\end{array}\right) \mathrm{A}_{s, i}\left(\mathcal{F}_{1}\right) \mathrm{F}_{k-s}\left(\mathcal{F}_{2}\right) .
$$

Analogously, we have $\left(T, a_{i}\right) \not \models \mathcal{F}$ if and only if $\left(T_{1}, a_{i}\right) \not \models \mathcal{F}_{1}$ and $T_{2} \not \models \mathcal{F}_{2}$, and, in this case, it holds that: $T \vDash \mathcal{F} \Leftrightarrow T_{1} \vDash \mathcal{F}_{1}$. Consequently,

$$
\mathrm{B}_{k, i}=\sum_{s=0}^{k}\left(\begin{array}{c}
k \\
k-s
\end{array}\right) \mathrm{B}_{s, i}\left(\mathcal{F}_{1}\right) \mathrm{F}_{k-s}\left(\mathcal{F}_{2}\right) .
$$

Finally, for $\mathrm{T}_{k}$ and $\mathrm{F}_{k}$, we have:

$$
\begin{aligned}
\mathrm{T}_{k} & =\sum_{s=0}^{k}\left(\begin{array}{c}
k \\
k-s
\end{array}\right)\left(\mathrm{T}_{s}\left(\mathcal{F}_{1}\right) \mathrm{T}_{k-s}\left(\mathcal{F}_{2}\right)+\mathrm{F}_{s}\left(\mathcal{F}_{1}\right) \mathrm{T}_{k-s}\left(\mathcal{F}_{2}\right)+\mathrm{T}_{s}\left(\mathcal{F}_{1}\right) \mathrm{F}_{k-s}\left(\mathcal{F}_{2}\right)\right), \\
\mathrm{F}_{k} & =\sum_{s=0}^{k}\left(\begin{array}{c}
k \\
k-s
\end{array}\right) \mathrm{F}_{s}\left(\mathcal{F}_{1}\right) \mathrm{F}_{k-s}\left(\mathcal{F}_{2}\right) .
\end{aligned}
$$

$-\mathcal{F}=\mathcal{F}_{1} \oplus \mathcal{F}_{2}$. Let $T=T_{1} \times T_{2}$ such that $T_{1} \in \Pi\left(X_{\mathcal{F}_{1}}\right),\left|T_{1}\right|=s$ and $T_{2} \in \Pi\left(X_{\mathcal{F}_{2}}\right),\left|T_{2}\right|=$ $k-s$. Now, the ordered coalition $T$ can contribute to $A_{k, i}$ in two ways. First, when $T_{1} \vDash \mathcal{F}_{1}$ and $T_{2} \vDash \mathcal{F}_{2}$ and $\left(T_{1}, a_{i}\right) \not \models \mathcal{F}_{1}$. Second, when $T_{1} \not \models \mathcal{F}_{1}$ and $T_{2} \not \models \mathcal{F}_{2}$ and $\left(T_{1}, a_{i}\right) \vDash \mathcal{F}_{1}$. Thus:

$$
\begin{aligned}
& \mathrm{A}_{k, i}=\sum_{s=0}^{k}\left(\begin{array}{c}
k \\
k-s
\end{array}\right)\left(\mathrm{B}_{s, i}\left(\mathcal{F}_{1}\right) \mathrm{T}_{k-s}\left(\mathcal{F}_{2}\right)+\mathrm{A}_{s, i}\left(\mathcal{F}_{1}\right) \mathrm{F}_{k-s}\left(\mathcal{F}_{2}\right)\right), \\
& \mathrm{B}_{k, i}=\sum_{s=0}^{k}\left(\begin{array}{c}
k \\
k-s
\end{array}\right)\left(\mathrm{B}_{s, i}\left(\mathcal{F}_{1}\right) \mathrm{F}_{k-s}\left(\mathcal{F}_{2}\right)+\mathrm{A}_{s, i}\left(\mathcal{F}_{1}\right) \mathrm{T}_{k-s}\left(\mathcal{F}_{2}\right)\right), \\
& \mathrm{T}_{k}=\sum_{s=0}^{k}\left(\begin{array}{c}
k \\
k-s
\end{array}\right)\left(\mathrm{T}_{s}\left(\mathcal{F}_{1}\right) \mathrm{F}_{k-s}\left(\mathcal{F}_{2}\right)+\mathrm{F}_{s}\left(\mathcal{F}_{1}\right) \mathrm{T}_{k-s}\left(\mathcal{F}_{2}\right)\right), \\
& \mathrm{F}_{k}=\sum_{s=0}^{k}\left(\begin{array}{c}
k \\
k-s
\end{array}\right)\left(\mathrm{T}_{s}\left(\mathcal{F}_{1}\right) \mathrm{T}_{k-s}\left(\mathcal{F}_{2}\right)+\mathrm{F}_{s}\left(\mathcal{F}_{1}\right) \mathrm{F}_{k-s}\left(\mathcal{F}_{2}\right)\right) .
\end{aligned}
$$

These four quantities are computed recursively in the function $S h^{N R}$, which is invoked in line 4 of Algorithm 1 (the pseudocode of $S h^{N R}$ can be found in Appendix A).

Note that $\mathrm{T}_{k}$ and $\mathrm{F}_{k}$ are only used to compute $\mathrm{A}_{k, i}$ and $\mathrm{B}_{k, i}$, which are then used in lines 7 and 9 of Algorithm 1 to compute $\phi^{N R}$. Having proved the correctness of computing $\mathrm{A}_{k, i}$ and $\mathrm{B}_{k, i}$, it remains to prove that the way they are used in lines 7 and 9 correctly computes $\phi^{N R}$. It suffices to prove this for a single rule $\mathcal{F} \rightarrow 1$, as the extension 
to multiple rules is straightforward in the MC-Net representation. To this end, some player $a_{i}$ can contribute to any ordered coalition $T$ counted in $\mathrm{A}_{k, i}$, or $\mathrm{B}_{k, i}$ (one of these quantities is zero), if it appears in a random permutation right after the players from $T$. The remaining players not occurring in $T$ can be arranged in $(m-k-1)$ ! ways (we have $\left|X_{\mathcal{F}}\right|=m$ ). In case $a_{i}$ contributes to $\mathrm{A}_{k, i}$, we have

$$
\phi_{i}^{N R}=\frac{1}{m !} \sum_{k=0}^{m-1}(m-k-1) ! \AA_{k, i} .
$$

In the second case $a_{i}$ contributes to $\mathrm{B}_{k, i}$; we get

$$
\phi_{i}^{N R}=-\frac{1}{m !} \sum_{k=0}^{m-1}(m-k-1) ! \mathrm{B}_{k, i} .
$$

These are exactly lines 7 and 9 of Algorithm 1 . This concludes the proof of correctness of Algorithm 1. In terms of runtime, it is clear from the pseudocodes (in Algorithm 1 and Appendix A) that the total number of operations is polynomial in the number of agents. This concludes the proof of Theorem 5.8.

\subsection{Computing the SB Value with Generalized Read-Once MC-Nets}

In this section, we discuss the computational properties of the generalized read-once MC-Net representation with respect to calculating the Sánchez-Bergantiños value (i.e., the SB value). We start our computational analysis with some observations about atomic formulas. First, observe that all players occurring in some $\mathcal{F}_{B A F}$ are indistinguishable. Furthermore, taking into account that each player can appear in at most one formula, $\phi^{S B}$ will assign to these players the same values. Similarly, for the case of $\mathcal{F}_{O A F}$, the $\mathrm{SB}$ value is the same for all players due to the symmetry axiom (see Example 3.4).

Note that the SB value involves a different notion of marginal contribution. Whereas computing quantities $\mathrm{T}_{k}$ and $\mathrm{F}_{k}$ remains the same, we need to redefine $\mathrm{A}_{k, i}$ and $\mathrm{B}_{k, i}$.

$$
\begin{aligned}
& \left.\mathrm{A}_{k, i, l}(\mathcal{F})=\left|T \in \mathcal{T}_{\mathcal{F}}:\right| T \mid=k, a_{i} \notin T, T \not \models \mathcal{F},\left(T, a_{i}\right)^{l} \vDash \mathcal{F}\right) \mid, \\
& \left.\mathrm{B}_{k, i, l}(\mathcal{F})=\left|T \in \mathcal{T}_{\mathcal{F}}:\right| T \mid=k, a_{i} \notin T, T \models \mathcal{F},\left(T, a_{i}\right)^{l} \not \models \mathcal{F}\right) \mid .
\end{aligned}
$$

The first quantity $\mathrm{A}_{k, i, l}(\mathcal{F})$ is the number of ordered coalitions of a given size $k$ for which adding player $a_{i}$ on position $l$ causes $\mathcal{F}$ to become satisfied. $\mathrm{B}_{k, i, l}$ is the number of coalitions in which adding the player $a_{i}$ on position $l$ causes $\mathcal{F}$ to become unsatisfied.

TheOREm 5.10. Given a generalized coalitional game $(N, \mathcal{G R})$, Algorithm 2 computes the $S B$ value $\left(\phi_{i}^{S B}\right.$ for all $\left.a_{i} \in N\right)$ in polynomial time.

Proof. As for $\mathrm{T}_{k}$ and $\mathrm{F}_{k}$, the computation is exactly the same as in the proof of Theorem 5.8 NR. Therefore, we only need to show how to calculate $\mathrm{A}_{k, i, l}$ and $\mathrm{B}_{k, i, l}$ in polynomial time. We will start from atomic formulas:

$-\mathcal{F}=\left\{a_{1}, \ldots, a_{r}\right\}$. In this case, $\mathrm{A}_{k, i, l}=(r-1)$ ! if $k=r-1, i \in X_{\mathcal{F}}$ and $l \in\{1, \ldots, r\}$, otherwise, $\mathrm{A}_{k, i, l}=0$. On the other hand, $\mathrm{B}_{k, i, l}=0$ for all $k, i$ and $l$.

$-\mathcal{F}=\left\langle a_{1}, \ldots, a_{r}\right\rangle$. Here, $\mathrm{A}_{k, i, l}=1$ if $k=r-1, i \in X_{\mathcal{F}}$ and $l=i$, otherwise $\mathrm{A}_{k, i, l}=0$. On the other hand, $\mathrm{B}_{k, i, l}$ is always equal to 0 .

In the next part of proof, we will use following proposition. 


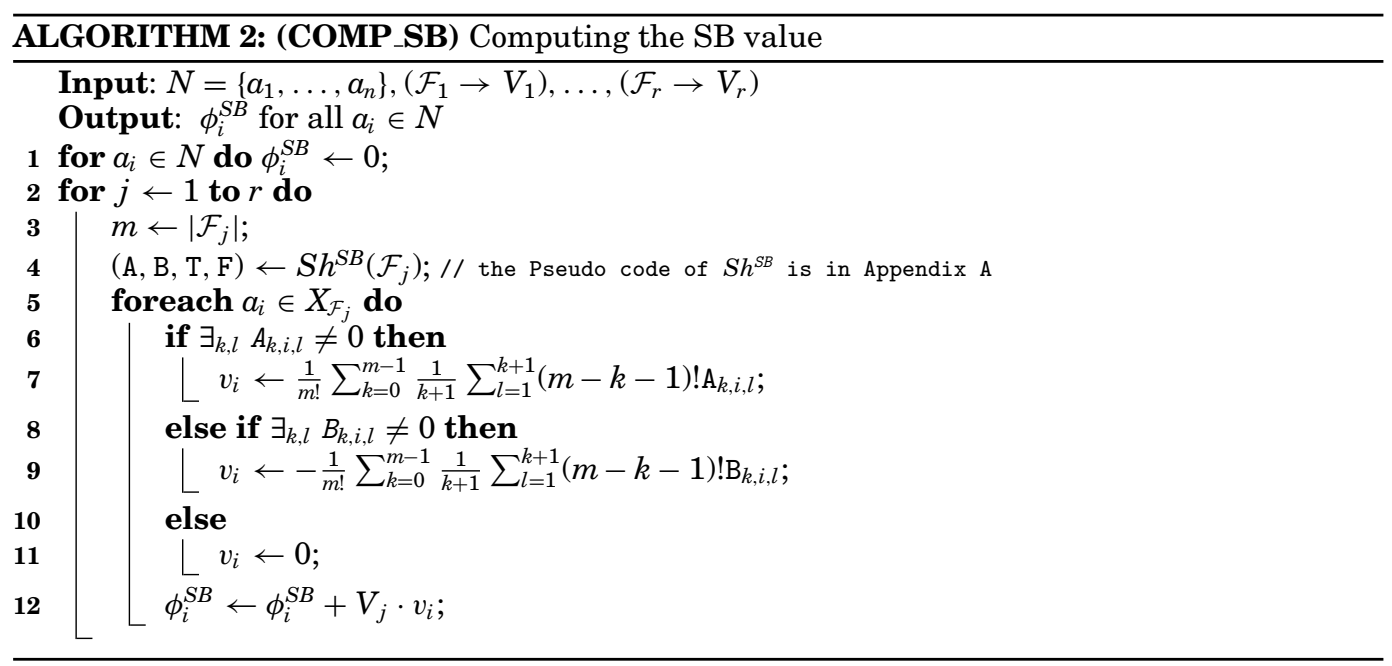

Proposition 5.11. The number of conflations of two ordered coalitions $T_{1}$ and $T_{2}$ such that s players from $T_{2}$ go before the lth member of $T_{1}$ equals

$$
\left(\begin{array}{c}
l+s-1 \\
s
\end{array}\right)\left(\begin{array}{c}
\left(\left|T_{1}\right|-l+1+1\right)+\left(\left|T_{2}\right|-s\right)-1 \\
\left|T_{2}\right|-s
\end{array}\right) .
$$

Proof. Simply use combination with repetition twice.

Next, we show how to compute $\mathrm{A}_{k, i}, \mathrm{~B}_{k, i}$ for each of the possible logical connective (i.e., $\neg, \wedge, \vee$ and $\oplus$ ) separately. We will assume that $a_{i} \in X_{\mathcal{F}_{1}}$.

$-\mathcal{F}=\neg \mathcal{F}_{1}$. Here, the negation causes a swap between sets. That is, we have $\mathrm{A}_{k, i, l}(\mathcal{F})=$ $\mathrm{B}_{k, i, l}\left(\mathcal{F}_{1}\right)$ and $\mathrm{B}_{k, i, l}(\mathcal{F})=\mathrm{A}_{k, i, l}\left(\mathcal{F}_{1}\right)$ for all $k, i, l$.

$-\mathcal{F}=\mathcal{F}_{1} \wedge \mathcal{F}_{2}$. Let $T=T_{1} \times T_{2}$, where $T_{1} \in \Pi\left(X_{\mathcal{F}_{1}}\right),\left|T_{1}\right|=s$ and $T_{2} \in \Pi\left(X_{\mathcal{F}_{2}}\right),\left|T_{2}\right|=$ $k-s$. We have $\left(T, a_{i}\right)^{l} \vDash \mathcal{F}$ if and only if $\left(T_{1}, a_{i}\right)^{m} \vDash \mathcal{F}_{1}$ for each $m \leq l$ when $l-m$ members of coalition $T_{2}$ go before the $m$ th member of coalition $T_{1}$ during the conflation operation and $T_{2} \vDash \mathcal{F}_{2}$. In this case, $T \not \models \mathcal{F}$ if and only if $T_{1} \not \models \mathcal{F}_{1}$. Consequently, using Proposition 5.11, we get

$$
\mathrm{A}_{k, i, l}=\sum_{s=0}^{k} \sum_{m=1}^{s+1}\left(\begin{array}{l}
l-1 \\
l-m
\end{array}\right)\left(\begin{array}{c}
k-l+1 \\
k-l+m-s
\end{array}\right) \mathrm{A}_{s, i, m}\left(\mathcal{F}_{1}\right) \mathrm{T}_{k-s}\left(\mathcal{F}_{2}\right)
$$

Furthermore, we have $T \vDash \mathcal{F}$ if and only if $T_{1} \vDash \mathcal{F}_{1}$ and $T_{2} \vDash \mathcal{F}_{2}$. Additionally in this case, it holds that: $\left(T, a_{i}\right)^{l} \not \models \mathcal{F} \Leftrightarrow\left(T_{1}, a_{i}\right)^{m} \not \models \mathcal{F}_{1}$ for $m \leq l$, when $l-m$ members of coalition $T_{2}$ go before the $m$ th member of coalition $T_{1}$ during the conflation operation and $T_{2} \not \models \mathcal{F}_{1}$. Again, using Proposition 5.11, we get

$$
\mathrm{B}_{k, i, l}=\sum_{s=0}^{k} \sum_{m=1}^{s+1}\left(\begin{array}{l}
l-1 \\
l-m
\end{array}\right)\left(\begin{array}{c}
k-l+1 \\
k-l+m-s
\end{array}\right) \mathrm{B}_{s, i, m}\left(\mathcal{F}_{1}\right) \mathrm{T}_{k-s}\left(\mathcal{F}_{2}\right)
$$


$-\mathcal{F}=\mathcal{F}_{1} \vee \mathcal{F}_{2}$. Once again let $T=T_{1} \times T_{2}$, where $T_{1} \in \Pi\left(X_{\mathcal{F}_{1}}\right),\left|T_{1}\right|=s$ and $T_{2} \in$ $\Pi\left(X_{\mathcal{F}_{2}}\right),\left|T_{2}\right|=k-s$. We know that $T \not \models \mathcal{F}$ if and only if $T_{1} \not \models \mathcal{F}_{1}$ and $T_{2} \not \models \mathcal{F}_{2}$. Furthermore, in this case, it holds that $\left(T, a_{i}\right)^{l} \vDash \mathcal{F} \Leftrightarrow\left(T_{1}, a_{i}\right)^{m} \vDash \mathcal{F}_{1}$ for $m \leq l$. Thus,

$$
\mathrm{A}_{k, i, l}=\sum_{s=0}^{k} \sum_{m=1}^{s+1}\left(\begin{array}{l}
l-1 \\
l-m
\end{array}\right)\left(\begin{array}{c}
k-l+1 \\
k-l+m-s
\end{array}\right) \mathrm{A}_{s, i, m}\left(\mathcal{F}_{1}\right) \mathrm{F}_{k-s}\left(\mathcal{F}_{2}\right) .
$$

Analogously, we have $\left(T, a_{i}\right)^{l} \not \models \mathcal{F}$ if and only if $\left(T_{1}, a_{i}\right)^{m} \not \models \mathcal{F}_{1}$ for $m \leq l$ when $l-m$ members of coalition $T_{2}$ go before the $m$ th member of coalition $T_{1}$ during the conflation operation, and $T_{2} \not \models \mathcal{F}_{2}$. Furthermore, in this case, it holds that: $T \vDash \mathcal{F} \Leftrightarrow T_{1} \vDash \mathcal{F}_{1}$. Consequently,

$$
\mathrm{B}_{k, i, l}=\sum_{s=0}^{k} \sum_{m=1}^{s+1}\left(\begin{array}{l}
l-1 \\
l-m
\end{array}\right)\left(\begin{array}{c}
k-l+1 \\
k-l+m-s
\end{array}\right) \mathrm{B}_{s, i, m}\left(\mathcal{F}_{1}\right) \mathrm{F}_{k-s}\left(\mathcal{F}_{2}\right) .
$$

$-\mathcal{F}=\mathcal{F}_{1} \oplus \mathcal{F}_{2}$. Let $T=T_{1} \times T_{2}$ such that $T_{1} \in \Pi\left(X_{\mathcal{F}_{1}}\right),\left|T_{1}\right|=s$ and $T_{2} \in \Pi\left(X_{\mathcal{F}_{2}}\right),\left|T_{2}\right|=$ $k-s$. Now, the ordered coalition $T$ can contribute to $A_{k, i, l}$ in two ways. First, when $T_{1} \vDash \mathcal{F}_{1}$ and $T_{2} \vDash \mathcal{F}_{2}$ and $\left(T_{1}, a_{i}\right)^{m} \not \models \mathcal{F}_{1}$. Second, when $T_{1} \not \models \mathcal{F}_{1}$ and $T_{2} \not \models \mathcal{F}_{2}$ and $\left(T_{1}, a_{i}\right)^{m} \vDash \mathcal{F}_{1}$, so

$$
\begin{aligned}
& \mathrm{A}_{k, i, l}=\sum_{s=0}^{k} \sum_{m=1}^{s+1}\left(\begin{array}{l}
l-1 \\
l-m
\end{array}\right)\left(\begin{array}{c}
k-l+1 \\
k-l+m-s
\end{array}\right)\left(\mathrm{B}_{s, i, m}\left(\mathcal{F}_{1}\right) \mathrm{T}_{k-s}\left(\mathcal{F}_{2}\right)+\mathrm{A}_{s, i, m}\left(\mathcal{F}_{1}\right) \mathrm{F}_{k-s}\left(\mathcal{F}_{2}\right)\right) . \\
& \mathrm{B}_{k, i, l}=\sum_{s=0}^{k} \sum_{m=1}^{s+1}\left(\begin{array}{l}
l-1 \\
l-m
\end{array}\right)\left(\begin{array}{c}
k-l+1 \\
k-l+m-s
\end{array}\right)\left(\mathrm{B}_{s, i, m}\left(\mathcal{F}_{1}\right) \mathrm{F}_{k-s}\left(\mathcal{F}_{2}\right)+\mathrm{A}_{s, i, m}\left(\mathcal{F}_{1}\right) \mathrm{T}_{k-s}\left(\mathcal{F}_{2}\right)\right) .
\end{aligned}
$$

These two quantities (together with $\mathrm{T}_{k}$ and $\mathrm{F}_{k}$ ) are computed recursively in the function $S h^{S B}$ which is invoked in line 4 of Algorithm 2 (the pseudocode of $S h^{S B}$ can be found in Appendix A).

Note that $\mathrm{T}_{k}$ and $\mathrm{F}_{k}$ are only used to compute $\mathrm{A}_{k, i, l}$ and $\mathrm{B}_{k, i, l}$, which are then used in lines 7 and 9 of Algorithm 2 to compute $S h^{S B}$. Having proved the correctness of computing $\mathrm{A}_{k, i, l}$ and $\mathrm{B}_{k, i, l}$, it remains to prove that the way they are used in lines 7 and 9 correctly computes $S h^{S B}$. It suffices to prove this for a single rule $\mathcal{F} \rightarrow 1$, as the extension to multiple rules is straightforward in the MC-Net representation. To this end, some player $a_{i}$ can contribute to any ordered coalition $T$ counted in $\mathrm{A}_{k, i, l}$, or $\mathrm{B}_{k, i, l}$, if it appears in a permutation right after the $(l-1)$ th player from $T$. The remaining players (i.e., those not belonging to $T)$ can be arranged in $(m-k-1)$ ! ways (we have $\left|X_{\mathcal{F}}\right|=m$ ). In case $a_{i}$ contributes to $\mathrm{A}_{k, i, l}$, we have

$$
\phi_{i}^{S B}=\frac{1}{m !} \sum_{k=0}^{m-1} \frac{1}{k+1} \sum_{l=1}^{k+1}(m-k-1) ! \mathrm{A}_{k, i, l} .
$$

In the second case, when $a_{i}$ contributes to $\mathrm{B}_{k, i, l}$, we get

$$
\phi_{i}^{S B}=-\frac{1}{m !} \sum_{k=0}^{m-1} \frac{1}{k+1} \sum_{l=1}^{k+1}(m-k-1) ! \mathrm{B}_{k, i, l} .
$$


These are exactly lines 7 and 9 of Algorithm 2. This concludes the proof of correctness of Algorithm 2. In terms of runtime, it is clear from the pseudocodes (in Algorithm 2 and Appendix A) that the total number of operations is polynomial in the number of agents. This concludes the proof of Theorem 5.10.

Finally, it is worth making a few remarks about the problem of generating a representation of a game in our framework. We know that given a representation of a game in our framework, we can, for example, compute the NR and SB values in polynomial time. But how hard is it to come up with a representation of a game in our framework?

Suppose we are given a black box representation of a characteristic function, that is, an oracle for the function that can be queried in unit time to determine the value of any ordered coalition. Then, it is not hard to see that it will require time exponential in the number of players to produce a representation of it in our framework. This is because we cannot avoid having to query the oracle for the value of every possible ordered coalition, and there are clearly exponentially many of them.

Another obvious question is whether other representations can easily be "translated" to our representation. Here, we have better news. For example, a representation of a game in Ieong and Shoham's MC-Nets representation can be transformed into our representation in polynomial time: MC-Net rules correspond directly to rules in our framework requiring only basic atomic formulae (BAFs). In the same way, the subgraph representation introduced by Deng and Papadimitriou [1994] (which is, in fact, a special case of the MC-Net representation) can also be translated into our framework in polynomial time. Each edge of the graph translates to a rule with a BAF containing only two players.

It is worth noting that we are not aware of any other attempts in the literature to develop representations for generalized coalitional games. If such were developed, then it would be interesting to consider the problem of translating between representations.

\subsection{Sample Application: Computing Generalized Game-Theoretic Betweenness Centrality}

As mentioned in the introduction, it is possible to use the game-theoretic solution concepts, including the NR and SB values, to measure centrality of nodes in networks [del Pozo et al. 2011]. In this section, we show how to extend the classic notion of betweenness centrality to generalized game-theoretic betweenness centrality which builds upon generalized characteristic function games and the NR and SB values. After that, we show that it is possible to use generalized read-once MC-Nets to compute the new centrality in time polynomial in the number of shortest paths in the network.

Betweenness centrality, independently introduced by Anthonisse [1971] and Freeman [1979], is one of the most important centrality measures. It quantifies the ability of a node to control the flow in the network. The first step to construct it is to calculate the number of all shortest paths between any pair of nodes in the network. Then, betweenness centrality of node $v$ is the proportion of shortest paths between any pair of nodes in the network that traverse through $v$.

While betweenness centrality focuses on the role in the network played by individual nodes, often it is important to consider the role played by groups of nodes. To this end, Everett and Borgatti [1999] introduced the concept of group centrality and, in particular, the concept of group betweenness centrality. Essentially, this measure works in exactly the same way as conventional betweenness centrality but now the proportion of shortest paths that traverse through a given group of 
nodes, not a single node, is calculated. An interesting version of group betweenness centrality was proposed by Puzis et al. [2007]. Their measure, called path betweenness centrality, evaluates sequences of nodes and counts the proportion of shortest paths that traverse along a given sequence of nodes in an appropriate order. Formally:

Definition 5.12. Given a graph $G=(V, E)$, we define the set of all ordered coalitions of nodes in the graph, that is, $\mathcal{T}=\bigcup_{C \in 2^{v}} \Pi(C)$. The path betweenness centrality of the ordered group of nodes $T$ is defined as a function $c_{p b}: \mathcal{T} \rightarrow \mathbb{R}$ such that:

$$
c_{p b}(T)=\sum_{\substack{s \notin T \\ t \notin T}} \frac{\sigma_{s t}(T)}{\sigma_{s t}},
$$

where $\sigma_{s t}$ denotes the number of all shortest paths from $s$ to $t$ and $\sigma_{s t}(T)$ is the number of shortest paths from $s$ to $t$ passing through all nodes from $T$ (if $s \in T$ or $t \in T$ then $\sigma_{s t}(T)=0$ ) such that the order of visited nodes is maintained (for path $p$ we have $T \subseteq \underline{\subseteq}$ ).

Similarly to other game-theoretic extension of classic centrality measures recently considered in the literature [Michalak et al. 2013a, 2013b; Narayanam et al. 2014; Skibski et al. 2014], this function, that assigns values to all ordered groups (coalitions) of nodes, can be considered to be a value function, that is, in our case, the generalized characteristic function. Now, the NR and SB values computed for this function become the generalized game-theoretic betweenness centrality. ${ }^{6}$

Definition 5.13. The generalized game-theoretic betweenness centrality is a triple $\left(G, c_{p b}, \phi\right)$, where $c_{p b}$ is the path betweenness centrality, the value of each node $v \in V$ is given by $\phi_{v}\left(c_{p b}\right)$, and $\phi$ is the NR value $\left(\phi^{N R}\right)$ or SB value $\left(\phi^{S B}\right)$.

Interestingly, some game-theoretic centrality measures can be computed in polynomial time in the size of the network, that is, in the number of nodes and edges [Szczepanski et al. 2014; Tarkowski et al. 2014]. While we did not obtain such a positive result for the generalized game-theoretic betweenness centrality, we will now show that it is possible to compute it in time polynomial in the number of shortest paths in the network and with the help of our generalized read-once MC-Nets.

In particular, given a network $G=(V, E)$, Algorithm 3 builds, for each shortest path in the network, a corresponding generalized read-once rule. More specifically, after initialization, we iterate over all shortest paths in the network. For each shortest path, in line 7 , we initialize the formula $\mathcal{F}$ with ordered atomic formula corresponding to the sequence of all the nodes on the path. Next, in line 8 , we add a conjunction that ensures that no other nodes can appear in an ordered coalition that satisfies our formula.

The size of the set $\mathcal{G} \mathcal{R}$ constructed by this algorithm equals to the number of shortest paths in the graph and can be exponential in the number of vertices. However, in many graphs we have $|\mathcal{G R}| \ll \mathcal{T}$, which makes this algorithm much more efficient than computing this measure directly using Eq. (9) or Eq. (12) for $\phi^{N R}$ and $\phi^{S B}$, respectively.

\footnotetext{
${ }^{6}$ We note that the game-theoretic betweenness centrality was introduced by Szczepański et al. [2012, 2014]_this measure, however, does not account for the order of players.
} 


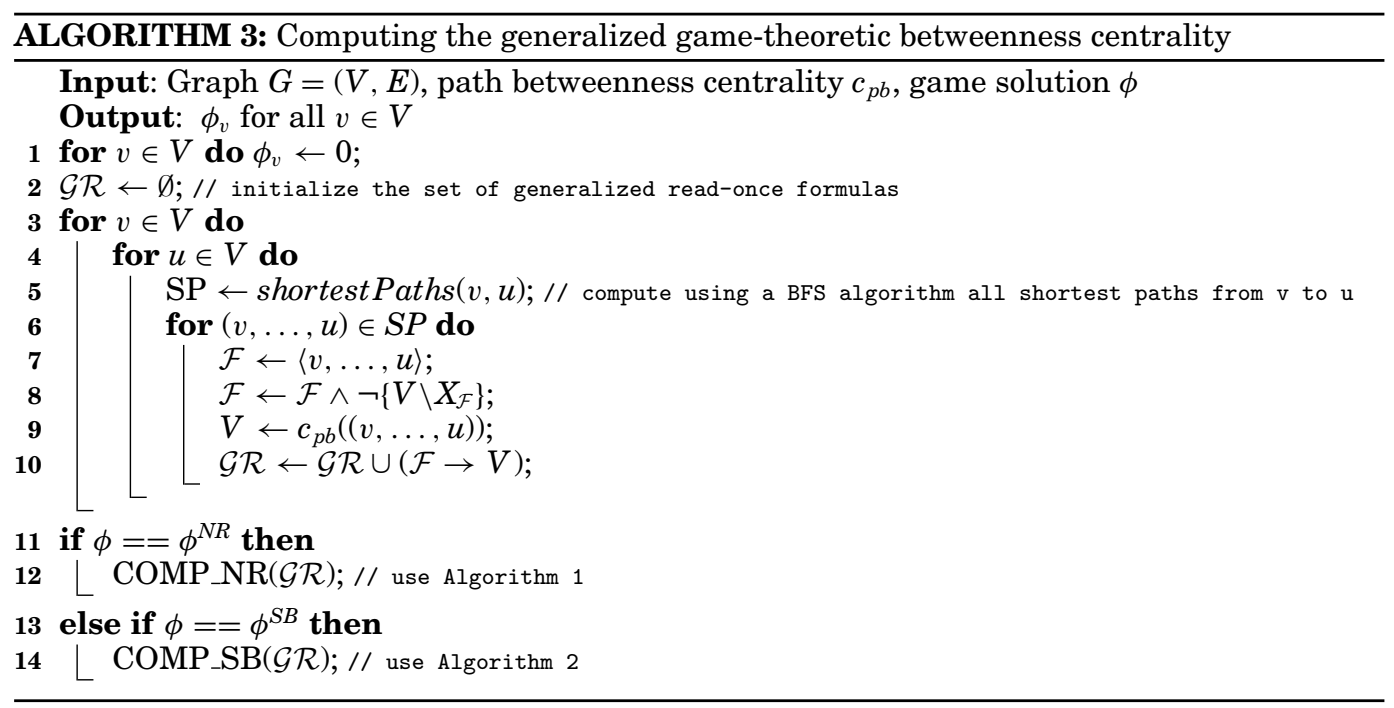

\section{CONCLUSIONS}

Generalized characteristic function games are attracting increasing interest in the literature due to their manifold potential applications. In this article we considered both the implementational and computational aspects of these games. In particular, building upon the mechanism for the Shapley value by Dasgupta and Chiu [1998], we proposed the first mechanism to implement two values for generalized characteristic function games: the Nowak-Radzik value and the Sánchez and Bergantiños value. Furthermore, to facilitate efficient computations supporting this mechanism, we proposed the Generalized Marginal-Contribution Nets representationthe first computationally convenient formalism to model games with ordered coalitions.

Whereas this work can be extended in various directions, two of them seem to be particularly interesting: (1) it would be interesting to consider representation formalisms other than MC-Nets, such as Algebraic Decision Diagrams Aadithya et al. [2011] and Sakurai et al. [2011], to model generalized characteristic function games, (2) it remains an open question whether it is possible to implement the values for the generalized characteristic function games by extending the mechanism of Pérez-Castrillo's and Wettstein's [2001] for the Shapley value. As mentioned in Section 2, this mechanism has two advantages over the mechanism by Dasgupta and Chiu [1998] (and, by extension, over our mechanism). First, the implementation is not in expected terms since the Shapley value emerges in all equilibria. Second, it does not require any a priori randomization of the order in which players make their moves. Nevertheless, the mechanism by Pérez-Castrillo's and Wettstein hinges upon the balanced contribution property which is not satisfied by the NR value. As a result, at present, it remains unknown whether it is possible to implement this value in such a spirit. Some hope that it may be possible is given by the extensions of the original mechanism by Pérez-Castrillo and Wettstein proposed by Ju and Wettstein [2009]. While these extensions deal only with characteristic function games, they implement two solution concepts that do not meet the balanced contribution property: the equal surplus value (see van den Brink and Funaki [2009]) and the consensus value (see Ju and Wettstein [2007]). 


\section{APPENDIXES}

\section{A. The Recursive Functions}

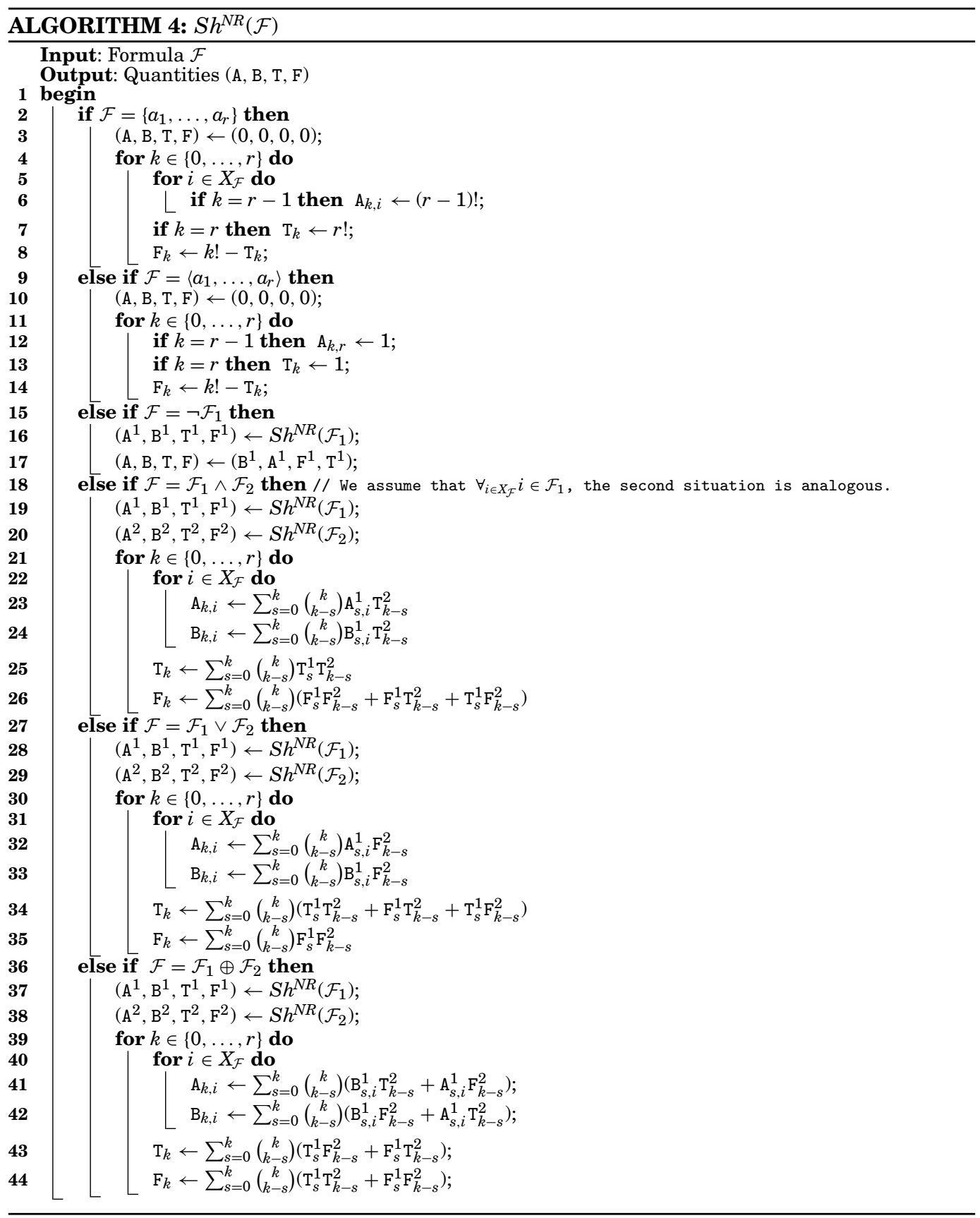




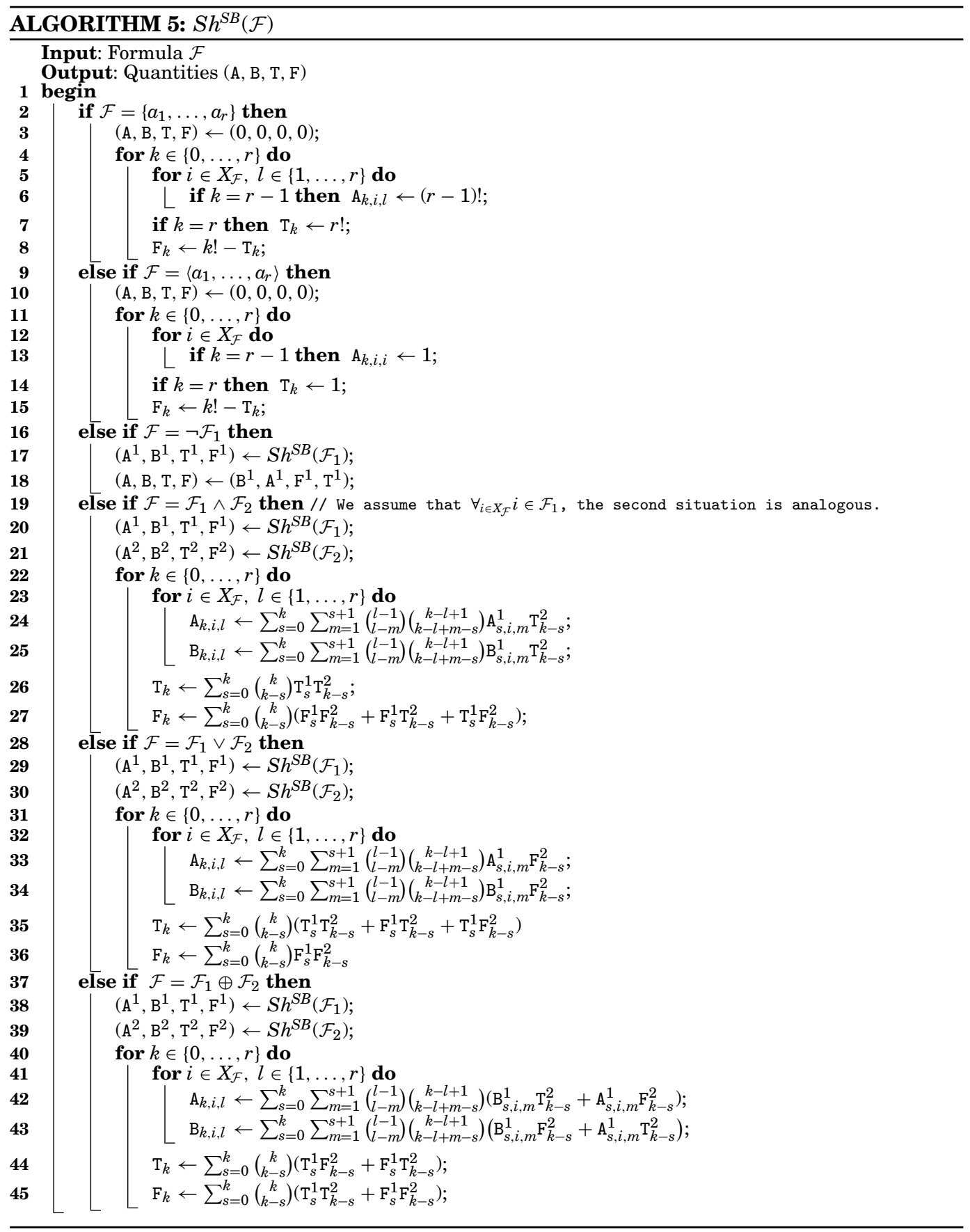




\title{
B. Main Notation Used in the Article
}

\author{
Players, coalitions, permutations \\ $N \quad$ The set of players. \\ $n \quad$ Cardinality of set $N$. \\ $a_{i} \quad$ A player in $N$. \\ $N_{-i} \quad$ The set of players $N$ without player $a_{i}$. \\ $C, D \quad$ A coalition. \\ $S, T \quad$ An ordered coalition. \\ $(T, S)^{k} \quad$ The ordered coalition that results from inserting $S$ at the $k$ th position \\ in $T$. \\ $\left(T, a_{i}\right) \quad$ The ordered coalition that results from inserting $a_{i}$ at the end of $T$. \\ An extension of the notion of a subset to ordered sets (see \\ Definition 3.1). \\ $\Pi(C)$ \\ $\pi$ \\ $\operatorname{inv}(\pi)$ \\ $\pi(C)$ \\ $v$ \\ $v_{g}$ \\ $v_{g, \pi}$ \\ $(N, v)$ \\ $\left(N, v_{g}\right)$ \\ $\left(N, \bar{v}_{g}\right)$ \\ $C_{\overleftarrow{\pi}(i)}$ \\ $\Delta_{v}\left(C_{\overleftarrow{\pi}(i)}, a_{i}\right)$ \\ $\Delta_{v_{g}}^{N R}\left(T, a_{i}\right)$ \\ $\Delta_{v_{g}}^{S B}\left(T, a_{i}\right)$ \\ $\Delta_{\bar{v}_{g}}\left(C_{\overleftarrow{\pi}(i)}, a_{i}\right)$ \\ $\phi_{i}(N, v)$ \\ $\phi_{i}^{N R}\left(N, v_{g}\right)$ \\ $\phi_{i}^{S B}\left(N, v_{g}\right)$ \\ $\mathbb{E}[\cdot]$ \\ $d_{i}$ \\ $O D C G_{\pi}^{N R / S B}$ \\ $H_{i}$
$h_{i}$
$\sigma_{\pi i}^{N R / S B}$
$M_{h, \pi}^{N R}(D)$ \\ $\mathcal{P}_{\pi}^{h}(i, j)$ \\ The set of all possible permutations of the players in $C$. \\ A permutation. \\ The inverse of $\pi$. \\ The ordered coalition which consists of all the players in $C$ and which \\ is ordered according to $\pi$. \\ Value functions, games, solution concepts \\ The characteristic function. \\ The generalized characteristic function. \\ The characteristic function, where $v_{g, \pi}(C)=v_{g}(\pi(C))$. \\ A coalitional game in a characteristic function form. \\ A coalitional game in a generalized characteristic function form. \\ The average game for $v_{g}$ ( $\bar{v}_{g}$ has the characteristic function form). \\ A coalition that consists of all the players that are in permutation $\pi$ \\ before $a_{i}$. \\ The marginal contribution of player $a_{i}$ to $C$ in the Shapley value \\ (Eq. (5)). \\ The marginal contribution of player $a_{i}$ to $T$ in the NR value. \\ The marginal contribution of player $a_{i}$ to $T$ in the SB value (Eq. (10)). \\ The marginal contribution of player $a_{i}$ to $C$ in the SB value (Eq. (5)). \\ The Shapely value of player $a_{i}$ in game $(N, v)$. \\ The Radzik-Nowak value of player $a_{i}$ in game $\left(N, v_{g}\right)$. \\ The Sánchez-Bergantiños value of player $a_{i}$ in game $\left(N, v_{g}\right)$. \\ The expectation operator. \\ $O D C G^{N R}$ and $O D C G^{S B}$ mechanisms \\ A "demand" made by player $a_{i}$. \\ The subgame for the NR value and the SB value, respectively, given \\ $\pi$. \\ The set of all histories that $a_{i}$ can face. \\ A history in $H_{i}$. \\ The strategy of player $a_{i}$ in $O D C G_{\pi}^{N R / S B}$. \\ The maximum payoff that coalition $D$ can obtain for itself if it is al- \\ lowed to optimally choose a set of new members $C$ from the players \\ that precede $a_{i}$. \\ A linear program.
}

$\underset{\mathcal{F}}{\left(z \wedge \neg z^{\prime}\right)} \rightarrow V$

\section{Representation}

Basic MC-Nets $z$ positive literals, $z^{\prime}$ positive literals,.

Formula 


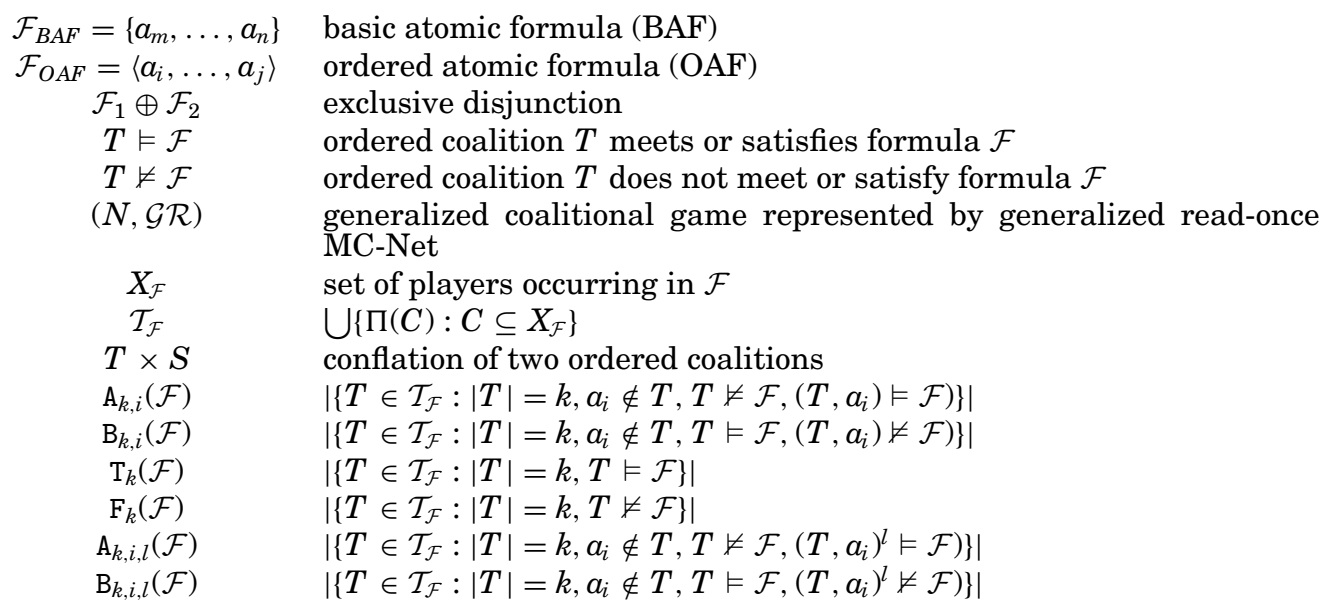

\section{ACKNOWLEDGMENTS}

The authors thank the ACM-TEAC anonymous reviewers and editors for their valuable comments, which greatly benefited the article.

\section{REFERENCES}

K. V. Aadithya, T. P. Michalak, and N. R. Jennings. 2011. Representation of coalitional games with algebraic decision diagrams. In Proceedings of the 12th International Conference on Autonomous Agents and Multiagent Systems (AAMAS). 1121-1122.

R. Amer, José M. Giménez, and A. Magãna. 2007. A ranking for the nodes of directed graphs based on game theory. In Proceedings in Applied Mathematics and Mechanics 7, 1.

R. Amer, J. M. Giménez Pradales, and A. Magaña Nieto. 2010. Nodes of directed graphs ranked by solutions defined on cooperative games. In Proceedings of the XXXII Congreso Nacional de Estadistica e Investigación Operativa. 1-20.

J. M. Anthonisse. 1971. The rush in a directed graph. Tech. Rep. BN 9/71.

Y. Bachrach, E. Markakis, A. D. Procaccia, J. S. Rosenschein, and A. Saberi. 2008. Approximating power indices. In Proceedings of the 7th International Conference on Autonomous Agents and Multi-Agent Systems (AAMAS). 943-950.

G. Bergantinños and E. Sánchez. 2001. Weighted Shapley values for games in generalized characteristic function form. An Official J. Spanish Soc. Statistics Oper. Res. 9, 1, 55-67.

R. B. Boppana and M. Sipser. 1990. The complexity of finite functions. In Handbook of Theoretical Computer Science (Volume A), MIT Press.

S. J. Brams, H. Mutlu, and S. L. Ramirez. 2006. Influence in terrorist networks: From undirected to directed graphs. Studies in Conflict Terrorism 29, 7.

J. Castro, D. Gómez, and J. Tejada. 2009. Polynomial calculation of the Shapley value based on sampling. Comput. Oper. Research 36, 5, 1726-1730.

G. Chalkiadakis, E. Elkind, and M. Wooldridge. 2011. Computational Aspects of Cooperative Game Theory. Morgan \& Claypool Publishers.

V. Conitzer and T. Sandholm. 2006. Complexity of constructing solutions in the core based on synergies among coalitions. Artifi. Intell. 170, 6, 607-619.

A. Dasgupta and Y. S. Chiu. 1998. On implementation via demand commitment games. Int. J. Game Theory 27, 2, 161-189.

M. del Pozo, C. Manuel, E. González-Arangüena, and G. Owen. 2011. Centrality in directed social networks. A game theoretic approach. Social Netw. 33, 3, 191-200.

X. Deng and C Papadimitriou. 1994. On the complexity of cooperative solution concepts. Math. Oper. Res. 19, 2, 257-266.

E. Elkind, L. Ann Goldberg, P. W. Goldberg, and M. Wooldridge. 2009. A tractable and expressive class of marginal contribution nets and its applications. Math. Logic Quart. 55, 4, 362-376.

R. A. Evans. 1996. Value, consistency, and random coalition formation. Games Econ. Behav. 12, 1, 68-80. 
M. G. Everett and S. P. Borgatti. 1999. The centrality of groups and classes . J. Math. Soc. 23, 3, $181-201$.

S. S. Fatima, M. Wooldridge, and N. R. Jennings. 2008. A linear approximation method for the Shapley value. Artif. Intell. J. 172, 14, 1673-1699.

L. C. Freeman. 1979. Centrality in social networks: Conceptual clarification. Soc. Netw. 1, 3, $215-239$.

G. Greco, E. Malizia, L. Palopoli, and F. Scarcello. 2009. On the complexity of compact coalitional games. In Proceedings of the International Joint Conference on Artificial Intelligence (IJCAI). 147-152.

F. Gul. 1989. Bargaining foundations of Shapley value. Econometrica 57, 1, 81-95.

S. Hart and Z. Levy. 1999. Efficiency does not imply immediate agreement. Econometrica 67, 4, 909-912.

S. Ieong and Y. Shoham. 2006. Marginal contribution nets: A complact representation scheme for coalitional games. In Proceedings of the ACM Conference on Electronic Commerce (ACM EC). 170-179.

Y. Ju and D. Wettstein. 2007. Bidding with coalitional externalities: A strategic approach to partition function form games. In European Meeting of the Econometric Society in Budapest, Hungary, 1-17.

Y. Ju and D. Wettstein. 2009. Implementing cooperative solution concepts: A generalized bidding approach. J. Econ. Theory 39, 2, 307-330.

D. Lehmann, L. I. O’Callaghan, and Y. Shoham. 2002. Truth revelation in approximately efficient combinatorial auctions. J. ACM 49, 5, 577-602.

H. K. Lentz, T. Selhorst, and I. M. Sokolov. 2012. Spread of infectious diseases in directed and modular metapopulation networks. Phys. Rev. E 85, 6, 066111.

I. Macho-Stadler, D. Pérez-Castrillo, and D. Wettstein. 2006. Efficient bidding with externalities. Games Econ. Behav. 57, 2, 304-320.

I. Macho-Stadler, D. Pérez-Castrillo, and D. Wettstein. 2010. Dividends and weighted values in games with externalities. Int. J. Game Theory 39, 1, 177-184.

T. P. Michalak, K. V. Aaditha, P. L. Szczepanski, B. Ravindran, and N. R. Jennings. 2013a. Efficient computation of the Shapley value for game-theoretic network centrality. J. Artif. Intell. Res. 46, 607-650.

T. P. Michalak, D. Marciniak, M. Szamotulski et al. 2010. A logic-based representation for coalitional games with externalities. In Proceedings of the 11th International Conference on Autonomous Agents and Multiagent Systems (AAMAS). 125-132.

T. P. Michalak, T. Rahwan, J. Sroka et al. 2009. On representing coalitional games with externalities. In Proceedings of the ACM Conference on Electronic Commerce. 11-20.

T. P. Michalak, T. Rahwan, P. L. Szczepanski et al. 2013b. Computational analysis of connectivity games with applications to the investigation of terrorist networks. In Proceedings of the International Joint Conference on Artificial Intelligence (IJCAI). (2013).

R. Narayanam, O. Skibski, H. Lamba, and T. P. Michalak. 2014. A Shapley value-based approach to determine gatekeepers in social networks with applications. In Proceedings of the 21st European Conference on Artificial Intelligence (ECAI'14).

A. Nowak and T. Radzik. 1994. The Shapley value for $n$-person games in generalized characteristic function form. Games Econ. Behav. 6, 1, 150-161.

N. Ohta, V. Conitzer, R. Ichimura, Y. Sakurai, A. Iwasaki, and M. Yokoo. 2009. Coalition structure generation utilizing compact characteristic function representations. In Proceedings of the Principles and Practice of Constraint Programming. 623-638.

N. Ohta, A. Iwasaki, M. Yokoo, and K. Maruono. 2006. A compact representation scheme for coalitional games in open anonymous environments. In Proceedings of the 20th Conference on Artificial Intelligence (AAAI). 509-514.

K. Hang Pham do and H. Norde. 2007. The Shapley value for partition function form games. Int. Game Theory Rev. 9, 2, 353-360.

D. Pérez-Castrillo and D. Wettstein. 2001. Bidding for the surplus: A non-cooperative approach to the Shapley value. J. Econ. Theory 100, 2, 274-294.

D. Pérez-Castrillo and D. Wettstein. 2005. Implementation of the ordinal Shapley value for a three-agent economy. Econ. Bull. 3, 48, 1-8.

D. Pérez-Castrillo and D. Wettstein. 2006. An ordinal Shapley value for economic environments. J. Econ. Theory 127, 1, 296-308.

Rami Puzis, Yuval Elovici, and Shlomi Dolev. 2007. Fast algorithm for successive computation of group betweenness centrality. Phys. Rev. E (Statistical, Nonlinear, and Soft Matter Physics) 76, 5.

I. Rahwan, S. Dsouza, A. Rutherford et al. 2013. Global manhunt pushes the limits of social mobilization. IEEE Comput. 46, 4, 68-75.

A. Rutherford, M. Cebrian, I. Rahwan et al. 2013. Targeted social mobilization in a global manhunt. PLOS One 8, 9, e74628. 
Y. Sakurai, S. Ueda, A. Iwasaki, S. Minato, and M. Yokoo. 2011. A compact representation scheme of coalitional games based on multi-terminal zero-suppressed binary decision diagrams. In Proceedings of the 12th International Conference on Principles of Practice in Multi-Agent Systems (AAMAS). 4-18.

E. Sánchez and G. Bergantiños. 1997. On values for generalized characteristic functions. OR Spektrum 19, 3, 229-234.

E. Sánchez and G. Bergantinños. 1999. Coalitional values and generalized characteristic functions. Math. Meth. Oper. Res. 49, 3, 413-433.

R. Serrano. 2004. Fifty years of the Nash program, 1953-2003. Tech. Rep. 2004-20, Brown University.

L. S. Shapley. 1953. A value for $n$-person games. In Contributions to the Theory of Games, volume II, H. W. Kuhn and A. W. Tucker (Eds.). 307-317.

Y. Shoham and K. Leyton-Brown. 2009. Multiagent Systems: Algorithmic, Game-Theoretic, and Logical Foundations. Cambridge University Press.

O. Skibski, T. P. Michalak, T. Rahwan, and M. Wooldridge. 2014. Algorithms for the Shapley and Myerson values in graph-restricted games. In Proceedings of the 14th International Conference on Autonomous Agents and Multiagent Systems - Volume 1 (AAMAS'14).

P. L. Szczepanski, T. P. Michalak, and T. Rahwan. 2012. A new approach to betweenness centrality based on the Shapley value. In Proceedings of the 11th International Conference on Autonomous Agents and Multiagent Systems - Volume 1 (AAMAS'12). 239-246.

P. L. Szczepanski, T. P. Michalak, and T. Rahwan. 2014. A game-theoretic measure of betweenness centrality. mimeo, University of Oxford.

M. Tarkowski, T. Michalak, T. Rahwan, E. Elkind, and M. Wooldridge. 2014. Game-theoretic network centrality measures: A critical survey. mimeo, University of Oxford.

R. van den Brink and Y. Funaki. 2009. Axiomatizations of a class of equal surplus sharing solutions for TU-games. Theory Dec. 67, 303-340.

R. van den Brink and Y. Funaki. 2010. Axiomatization and implementation of discounted Shapley values. Tinbergen institute discussion papers. Tinbergen Institute.

J. Vidal-Puga and G. Bergantiños. 2003. An implementation of the Owen value. Games Econ. Behav. 44, 2, 412-427.

M. Wooldridge and P. Dunne. 2006. On the computational complexity of coalitional resource games. Artif. Intell. 170, 835-871.

Received June 2013; revised May 2014; accepted July 2014 